\title{
HST observations of nuclear stellar disks ${ }^{\star} \star \star$
}

\author{
D. Krajnović and W. Jaffe
}

\begin{abstract}
Sterrewacht Leiden, Postbus 9513, 2300 RA Leiden, The Netherlands e-mail: davor@strw.leidenuniv.nl
\end{abstract}

Received 1 March 2004 / Accepted 11 August 2004

\begin{abstract}
We present observations of four nearby early-type galaxies with previously known nuclear stellar disks using two instruments on-board the Hubble Space Telescope. We observed NGC 4128, NGC 4612, and NGC 5308 with the Wide Field Planetary Camera 2, and the same three galaxies, plus NGC 4570, with the Space Telescope Imaging Spectrograph. We have detected a red nucleus in NGC 4128, a blue nucleus in NGC 4621, and a blue disk in NGC 5308. Additionally, we have discovered a blue disk-like feature with position angle $\sim 15^{\circ}$ from the major axis in NGC 4621. In NGC 5308 there is evidence for a blue region along the minor axis. We discovered a blue transient on the images of NGC 4128 at position 0 .' 14 west and 0.32 north from the nucleus. The extracted kinematic profiles belong to two groups: fast (NGC 4570 and NGC 5308) and kinematically disturbed rotators (NGC 4128 and NGC 4621). We report the discovery of a kinematically decoupled core in NGC 4128. Galaxies have mostly old (10-14 Gyr) stellar populations with large spread in metallicities (sub- to super-solar). We discuss the possible formation scenarios, including bar-driven secular evolution and the influence of mergers, which can explain the observed color and kinematic features.
\end{abstract}

Key words. galaxies: elliptical and lenticular, cD - galaxies: nuclei - galaxies: kinematics and dynamics galaxies: photometry - galaxies: stellar content - galaxies: evolution

\section{Introduction}

The decade of Hubble Space Telescope (HST) observations have revealed the existence of small scale nuclear stellar disks in early-type galaxies. This discovery was an important step in the long process of recognizing the complexity of early-type galaxies. Ground-based studies preceding the HST era, having lower resolution and polluted by typically $>1^{\prime \prime}$ seeing, already recognized two distinct classes of elliptical galaxies (Davies et al. 1983) that differed in photometric appearance - disky vs. boxy - and kinematic properties - rotationally vs. pressure supported - (Bender 1988; Bender et al. 1989). Follow-up studies discovered the existence of embedded stellar disks in elliptical and lenticular galaxies (Scorza \& Bender 1995; Seifert \& Scorza 1996). These disks, although similar to their counterparts in spiral and S0 galaxies, have smaller scale length and higher central surface brightness. They often do not follow the same exponential profiles, and are closer to $r^{1 / 4}$ profiles, reflecting formation in a different potential: dark halo potentials for disks in late-types, and bulge potentials for disks in earlytype galaxies (Scorza \& Bender 1995). The existence of the embedded disks also supports the idea of the morphological

$\star$ Appendix A is only available in electronic form at the CDS via anonymous ftp to cdsarc.u-strasbg.fr (130.79.128.5) or via http://cdsweb.u-strasbg.fr/cgi-bin/qcat?]/A+A/428/877

$\star \star$ Appendix B, Tables 2, 3, 5-8 and Figs. 1, 3-5, 8-11 are only available in electronic form at http://www. edpsciences.org connection between spiral, lenticular and elliptical galaxies (Kormendy \& Bender 1996).

The properties of the nuclear regions (inner few $100 \mathrm{pc}$ ) of early-type galaxies are, however, not easily accessible from the ground. High resolution imaging surveys with HST discovered small scale nuclear stellar disks in early type galaxies (Jaffe et al. 1994; van den Bosch et al. 1994; Lauer et al. 1995; Rest et al. 2001, hereafter R01). They were followed by detailed photometric and kinematic studies on a few individual objects, principally NGC 4342 (Scorza \& van den Bosch 1998; van den Bosch et al. 1998, hereafter BJM98), NGC 4570 (BJM98; Scorza \& van den Bosch 1998; van den Bosch \& Emsellem 1998), NGC 4594 (Burkhead 1986; Kormendy 1988; Emsellem et al. 1996), NGC 7332 (Fisher et al. 1994; Falcón-Barroso et al. 2004). A detailed study of early-type galaxies with kinematicaly distinct components (Carollo et al. 1997a,b) found photometric evidences for faint nuclear stellar disks in a number of dust free galaxies.

The next step was a search for embedded nuclear stellar disks in bulges of spiral galaxies. The high resolution studies of spiral galaxies with HST showed that a significant fraction of galaxies classified as early-type spirals have a rich variety of central properties, and show little evidence for $r^{1 / 4}$ law expected for smooth bulges (Carollo et al. 1998). Similarly, Balcells et al. (2003) found moderately large fraction $(34 \%)$ of nuclear bars or disks in their HST near-infrared survey of S0-Sbc galaxies. Using archival HST imaging, 
Pizzella et al. (2002) reported evidence for nuclear disks in three early-type spirals and concluded that the disks are restricted to S0 and unbarred spiral galaxies. Having in mind that nuclear stellar disks are detectable only when seen nearly edge on (Rix \& White 1990), they appear to be very common, perhaps universal, in flattened ellipticals and S0s.

The large fraction of detected nuclear disks in early-type galaxies (51\% in the R01 sample) presents the questions: how and when did the nuclear stellar disks form? Nuclear stellar disks are found in S0 and disky ellipticals, but they are not simple extensions of large scale disks to the center of the galaxy. Often there is clear photometric and kinematic evidences for double disk structures (van den Bosch et al. 1994; Scorza \& Bender 1995; Scorza \& van den Bosch 1998), where the double disk structures are represented by two morphologically separated disks, having different scale lengths, lying in nearly same plane, but possibly with different inclinations, and having an inner/outer separation radius between the disks. On the other hand, Erwin \& Sparke (2002) show evidences that some inner disks seen in edge-on galaxies could be bars mistaken for disks. In any case, the two dynamically different structures are not easily distinguished in all cases.

Inner disks are also found inside bars or rings (van den Bosch \& Emsellem 1998; Erwin \& Sparke 1999; Erwin et al. 2003). This is important for understanding their formation. Nuclear stellar disks could be the result of mergers in hierarchical galaxy formation scenario: accretion of gas during the merger which settles in the principal plane of the galaxy and then makes stars. On the other hand, disks could be formed from the galaxy material transported to the nucleus by a bar, or perhaps from a mixture of these processes, in which a bar fuels the center effectively with gas captured at some previous epoch. Whatever scenario we choose, it has to be consistent with the high metallicities seen in the disks (BJM98; Emsellem et al. 1996) as well as their blue colors (BJM98; Kormendy et al. 2002) implying younger stellar populations.

Nuclear stellar disks with their cold dynamical properties and high surface brightness provide an excellent measure of the central mass-to-light ratio, as well as of the mass of the central black hole (van den Bosch \& de Zeeuw 1996). A few studies used this to determine the mass of the back holes in galaxies with nuclear stellar disks (Kormendy et al. 1996a,b; Cretton \& van den Bosch 1999; Emsellem et al. 1999).

Early-type galaxies are also interesting for studying stellar populations. The absence of strong and continuous starformation as well as emission line gas makes it easier to investigate the formation history and the connection between the photometric morphology, dynamical structures and corresponding stellar populations.

In order to increase the available dataset and to investigate the dependencies between the kinematics and line-strengths, as well as to determine the mass of black holes, we obtained high resolution spectra of four galaxies known to have nuclear stellar disks from the R01 sample: NGC 4128, NGC 4570, NGC 4621 and NGC 5308. In addition, we also imaged three of the galaxies, except NGC 4570 which was thoroughly investigated in the previous studies (BJM98, Scorza \& van den Bosch 1998; van den Bosch \& Emsellem 1998). In this paper we present data
Table 1. The properties of sample galaxies.

\begin{tabular}{cccccccc}
\hline \hline Galaxy & Type & $M_{B}$ & $B-V$ & $v_{\text {rad }}$ & PA & $D$ & Scale \\
$(1)$ & $(2)$ & $(3)$ & $(4)$ & $(5)$ & $(6)$ & $(7)$ & $(8)$ \\
\hline NGC 4128 & $S 0$ & -19.89 & 1.02 & 2610 & 58 & 36.3 & 175.7 \\
NGC 4570 & $S 0$ & -20.39 & 0.97 & 1811 & 159 & 25.2 & 121.9 \\
NGC 4621 & $E$ & -20.49 & 0.97 & 524 & 165 & 7.3 & 35.3 \\
NGC 5308 & $E-S 0$ & -20.38 & 0.93 & 2299 & 60 & 31.9 & 154.8 \\
\hline
\end{tabular}

Notes - Column (1): galaxy name; Col. (2): morphological type; Col. (3): absolute $B$-magnitude; Col. (4): apparent $B-V$ color within the effective aperture in which half of the $B$-flux is emitted; Col. (5): radial velocity (cz) in $\mathrm{km} \mathrm{s}^{-1}$ corrected for LG infall onto Virgo; Col. (6): major axis position angle in degrees; Col. (7): distance in Mpc, as derived from radial velocity (Col. 5) using Hubble constant $H_{0}=72 \mathrm{~km} \mathrm{~s}^{-1} \mathrm{Mpc}^{-1}$ (Freedman et al. 2001); Col. (8): distance scale in pc $\operatorname{arcsec}^{-1}$. Values listed in Cols. 2-6 are taken from Lyon/Meudon Extragalactic Database (LEDA).

observed with two instruments on-board HST during Cycle 9 (Program ID 8667) and concentrate on the photometric and spectroscopic properties and dependencies. Dynamical modeling of the galaxies with the purpose of determining the masses of the central black holes will follow in a separate paper.

Section 2 presents broad band photometry, data reduction, isophotal analysis and color images. Section 3 deals with spectroscopic observations, data reduction, extraction of kinematics and measurements of line-strengths. Section 4 presents a discussion of the results for individual galaxies. The conclusions and summary of the work are presented in Sect. 5 .

\section{WFPC2 broad band imaging}

The Wide Field Planetary Camera 2 (WFPC2) observations (Biretta et al. 1996) included imaging in $V(F 555 W)$ and $B$ and $(F 450 \mathrm{~W})$. The general properties of the sample galaxies are presented in Table 1, the observations are summarized in Table 2 and the details of the filter properties are listed in Table 3. The centers of the galaxies were positioned on the PC CCD. The size of the PC CCD is $800 \times$ 800 pixels of $0{ }^{\prime} 0455 \times 00^{\prime} 0455$. All exposures were taken with the telescope in fine lock. In addition to newly acquired data, we used existing archival $I(F 814 \mathrm{~W})$ band images for NGC 4621 (Program ID 8212, PI Ajhar) and NGC 5308 (Program ID 5512, PI Faber). There were no $I$ band observations for NGC 4128 in the archive.

\subsection{Data reduction}

The images were reduced through the standard HST/WFPC2 pipeline. Upon request of the data, the On-The-Fly reprocessing system re-reduced the data using the best calibration files. The standard reduction steps include correction for analog to digital conversion error, bias and dark current subtraction and flat-fielding (for more description see Holtzman et al. 1995). Our observations were divided (CR-SPLIT) in two (per filter). To combine them and to remove the cosmic rays we used a set of IDL routines from The IDL Astronomy User's Library 
(Landsman 1993) ${ }^{1}$. The WFPC2 images (PC, WF2, WF3, and WF4 for both CR-SPLIT sections) are cross-correlated to determine a possible shift between the exposures, aligned and combined removing the cosmic rays using an IDL equivalent of the IRAF task CRREJ.

We then constructed color images: $B-I, V-I$ and $B-V$. To construct the color images we had to align the individual images very precisely. This was achieved by rotating the original images for the difference in the telescope orientation angle, sub-sampling pixels by a factor of six and crosscorrelating images to find the shift. After all shifts were applied, the images were rebinned to the original pixel size. Both images used for the construction of a color image were initially convolved with the PSF of the other image. The PSFs were constructed using Tiny Tim software (Krist \& Hook 2001). The raw counts of the images were converted into JohnsonCousins $B, V$ and $I$ magnitudes following the guidelines given by Holtzman et al. (1995), using the zero points as given by Dolphin $(2000)^{2}$ and iterating the calibration until convergence. Note that the iteration was not performed for the $B-I$ color images, because there were no published transformation for the $F 450 W$ filter using $B$ and $I$. We estimate our relative photometric accuracy to be $\approx 0.02 \mathrm{mag}$, while the absolute uncertainty is $\approx 0.05 \mathrm{mag}$ (and $\approx 0.1$ for $B-I$ color). In Figs. 1 and 2 we present WFPC2 observations, isophotal analysis, broadband and color images of three observed galaxies.

\subsection{Isophotal analysis}

In order to investigate the disky structure of the galaxies we used the IRAF task "ellipse" to perform isophotal fits to the light distributions. We measured the ellipticity and position angle of the isophotes, as a function of radius. The method (for a full description see Jedrzejewski 1987) first fits elliptical isophotes to a Fourier expansion of first and second order terms. The next step in the method is to measure the higher order terms of the Fourier expansion. The pure ellipse is given by the first two order terms in the expansion. Any non zero values of the higher order $(>2)$ terms means a deviation from the perfect ellipse. Peletier et al. (1990) and Goudfrooij et al. (1994) found that $\cos 3 \theta$ terms $\left(b_{3}\right)$ are sensitive to the presence of dust (as well as the difference between the higher order terms in different bands), while the $\cos 4 \theta$ terms $\left(b_{4}\right)$ describe the shape by distinguishing boxy $\left(b_{4}<0\right)$ from disky $\left(b_{4}>0\right)$ galaxies (e.g. Lauer 1985; Bender 1988). The isophotal parameters for three galaxies are shown in Fig. 1. Different studies (van den Bosch et al. 1994, R01) showed that although the isophote parameters can be fitted down to 0.03 , they are not reliable and only values $>0$.' 2 should be used for analysis.

The galaxies were selected on the basis of having a nuclear stellar disk and our isopohotal analysis agrees well with the R01 results. For all three galaxies the $b_{3}$ terms are consistent with being zero in the reliable range $(>0,2)$. The $b_{4}$ terms have clear disky deviations, but there are differences between the galaxies. NGC 4128 shows the smallest positive values

\footnotetext{
1 http://idlastro.gsfc.nasa.gov

${ }^{2}$ http://www.noao.edu/staff/dolphin/wfpc2_calib/
}

of $b_{4}$ coefficient, and is the only galaxy where $b_{4}$ becomes negative. It drops below zero at a radius of $\sim 3^{\prime \prime}$ from the center. The isophotes remain boxy in the rest of the investigated range $\left(3-10^{\prime \prime}\right)$. The fourth order term in NGC 4621 is positive in the investigated range, although it starts to decrease beyond $3^{\prime \prime}$. NGC 5308 shows the sharpest rise of the $b_{4}$ coefficient, but it drops to zero around $7^{\prime \prime}$ before it rises again. The analysis of photometric higher order terms suggests the nuclear stellar disks are separated from the large scale disks.

The coefficients of this isophotal analysis are onedimensional representations of $2 \mathrm{D}$ structures in the galaxies. In order to see more clearly the nuclear disks we constructed residual $B$ and $V$ images by subtracting a model galaxy from the original. The model galaxy was constructed using the IRAF task "bmodel". It had the same luminosity profile as the original galaxy, but it was constructed from perfect ellipses (first two terms in the Fourier expansion). The contour maps, shown in the middle row in Fig. 2, reveal the disky structure that is responsible for the existence of the higher order terms. These disky structures do not necessarily show the full disks, because some information on the disks is also contained in the ellipticity, which was subtracted by the model. However, to the first approximation, these structures are a good representation of the relatively faint nuclear stellar disks in the observed galaxies. The disks have various sizes between $2-5^{\prime \prime}$, corresponding to $150-600 \mathrm{pc}$, and are in a good agreement with the spatial extent of positive values of $b_{4}$ terms. The noise structure in Fig. 2 perpendicular to the disks (along the minor axis) is not real and is an artifact of subtracting a perfect elliptical structure from a very disky one.

\subsection{Broad-band color images}

We constructed several broad band images using our data and archival images as described in Sect. 2.1. In the bottom two rows of Fig. 2 we show $B-V$ and $B-I$ images for all three galaxies except for NGC 4128 which does not have archival I band images. These color images are color coded such that lighter shades indicate redder colors.

\subsection{1. $B-V$ color images}

The nuclei of the galaxies show different structures in the $B-V$ color. The nucleus of NGC 4128 is redder then the surrounding bulge, NGC 4621 has a convincingly blue nucleus, while NGC 5308 is rather uniform with a slightly bluer central pixel. The magnitudes measured on the constructed color images are presented in Table 4 . We measured the color within a small circular aperture (6 pixels in diameter) to point out the subtle differences in colors between different regions and features. The blue feature on the color image of NGC 4128 corresponds to a transient object detected in the galaxy. The nature of the transient is not known (see Appendix B for a detailed discussion). In NGC 4128 isochromes trace the nuclear disk, while in the case of NGC 5308 there is weak evidence of a blue disk outside $0 ! 3$ of the nucleus. The difference is $\approx 0.02$ mag. NGC 4621 is a special case different from both previous 


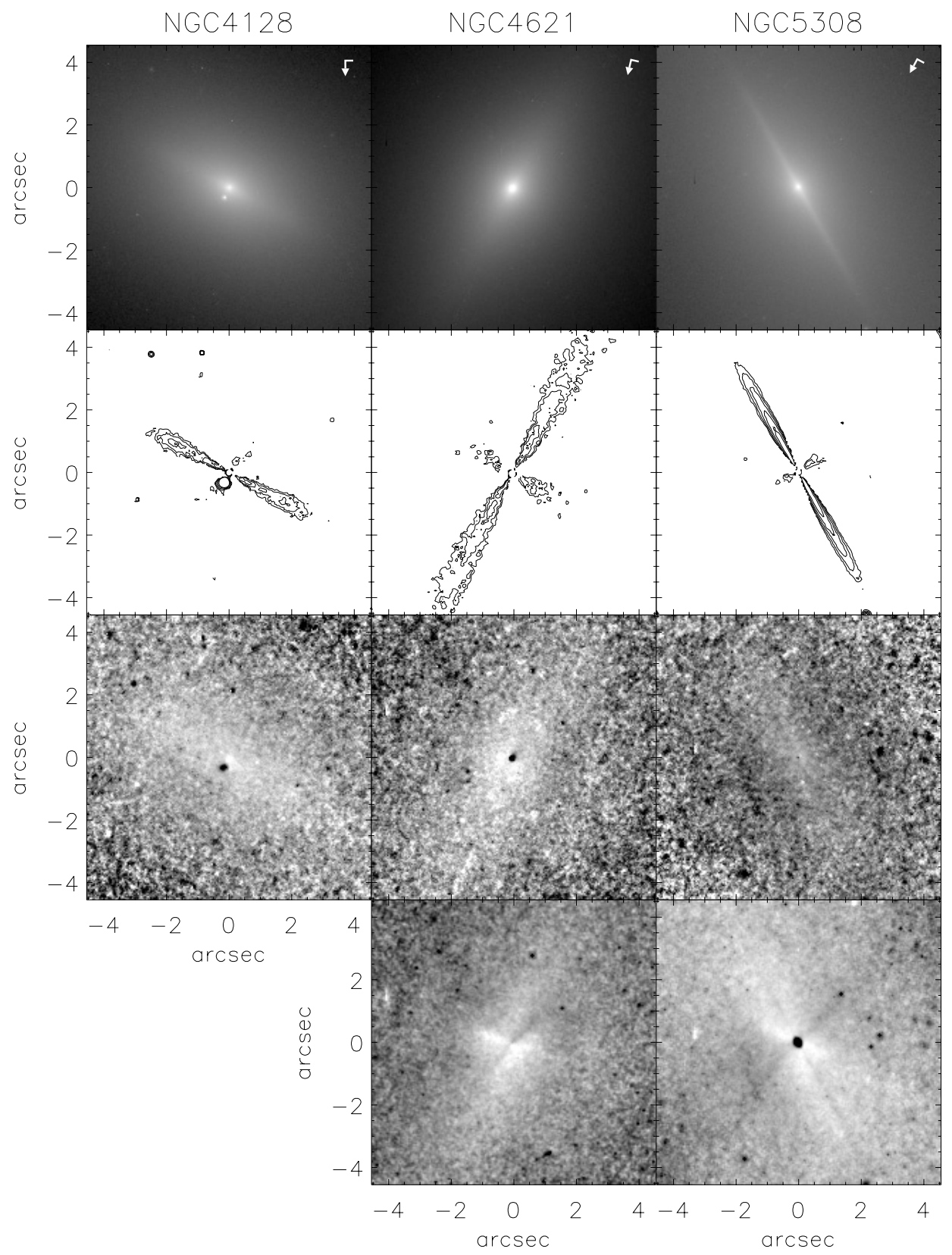

Fig. 2. WFPC2 observation of NGC 4128, NGC 4621, NGC 5308. First row: images in the $V(F 555 W)$ filter. The arrows and their associated dashes mark the North and East orientation of the images. Second row: residual stellar disks after subtraction of perfect elliptical model galaxy (in $V$ band). Contours were slightly smoothed and logarithmically scaled with steps of 0.5 mag. Third row: $B-V$ images, with grayscale such that brighter means redder. The blue feature next to the center of NGC 4128 is due to the transient and is discussed in Appendix B. The plotted range is (in magnitudes) 0.6 (black) to 1.0 (white) for NGC 4128, 0.5 (black) to 1.05 (white) for NGC 4621 , and 0.5 (black) to 0.98 (white) NGC 5308. Fourth row: $B-I$ images. The central blue feature on the NGC 5308 color map is an artifact of saturation and was not considered in the analysis. Range is from 1.93 (black) to 2.16 (white) for NGC 4621, and 1.1 (black) to 2.07 (white) for NGC 5308.

galaxies. Here we have a prominent blue nucleus on top of the red bulge. The other particularity of this galaxy is in the blue component that stretches southwards from the nucleus. The average difference between this component and the rest of the bulge is again $\approx 0.02$.

\subsection{2. $B, V-I$ color images}

A bigger color difference is seen in the $B-I$ and $V-I$ images which we constructed for NGC 4621 and NGC 5308.
The archival I-band images of NGC 4621 were of good quality, while images of NGC 5308 were saturated, and the very central parts of the images are not reliable.

The interesting blue feature southwards from the nucleus on the $B-I$ image of NGC 4621 is more prominent and we can quantify its extent. The color difference between the red bulgelike background and the blue patch is here $\approx 0.12 \mathrm{mag}$ in its extreme. The extent of the feature is $\approx 1$ '. 35 on each side of the nucleus, being somewhat bluer on the south side. The feature lies generally in the north-south direction making an angle of $15^{\circ}$ 
Table 4. Color in magnitudes measured within apertures of 6 pixels $(0,273)$ in diameter.

\begin{tabular}{cccccccc}
\hline \hline Galaxy & \multicolumn{2}{c}{$B-V$} & \multicolumn{2}{c}{$V-I$} & \multicolumn{2}{c}{$B-I$} \\
& center & average & center & $B F$ & bulge & $B F$ \\
$(1)$ & $(2)$ & $(3)$ & $(4)$ & $(5)$ & $(6)$ & $(7)$ \\
\hline NGC 4128 & 1.00 & 0.95 & - & - & - & - \\
NGC 4621 & 0.87 & 0.99 & 1.22 & 1.16 & 2.14 & 2.05 \\
NGC 5308 & 0.97 & 0.94 & - & - & - & - \\
\hline
\end{tabular}

Notes - Column (1): galaxy name; Col. (2): $B-V$ color measured at the center of the galaxies; Col. (3): $B-V$ color averaged over 8 apertures placed around center on a square grid centered on the nucleus with size $2 \times 14$ pixels $\left(\sim 1^{\prime \prime} 27\right)$, except for NGC 4128 where the blue feature next to the center was excluded; Col. (4): $V-I$ color measured at the center of galaxy; Col. (5): $V-I$ color measured at the blue feature $0{ }^{\prime} 90$ from the center; Col. (6): $B-I$ color measured at bulge of the galaxy; Col. (7): $B-I$ color measured at the blue feature $00^{\prime} 90$ from the center.

to the major axis. It is in no obvious plane of symmetry of the galaxy. The rest of the bulge is red, but a region of enhanced red color lies in the east-west direction. The angle between this reddest region and the major axis of about $103^{\circ}$, making the angle between the bluest and the reddest regions about $118^{\circ}$. The $V-I$ color image confirms this finding, also noticed by Wernli et al. (2002), hereafter WEC02, on their $V-I$ images. We postpone the discussion and interpretation of these features to Sect. 4.3 .

NGC 5308 is an equally interesting case. The center of the $I$ band image was saturated and the blue nucleus is an artifact. Generally, one has to be very careful in interpreting the saturated images. Aware of problems in dealing with saturated images, we used them to verify the faint suggestion from the $B-V$ images of the blue disk. We do not consider the central 0.5 of the $B-I$ and $V-I$ color images (the area somewhat larger than the blue dip in the images), but concentrate on the larger-scale features.

Along the minor axis of the galaxy, the $B-I$ color image also reveals blue regions on each side of the nucleus, as well as a red feature in the east-west direction. These features were not anticipated and we checked whether they are real or artifacts of saturation since they are close to the nucleus $\left(r<1^{\prime \prime}\right)$. We first constructed color image from unconvolved $F 4550 \mathrm{~W}$ and $F 814 \mathrm{~W}$ images. On the resulting color image, there was a hint of the east-west red feature. The other approach included deconvolution of the $B$ and $V$ images using Richardson-Lucy algorithm with 20 iterations. The resulting images were convolved with the PSF of the $I$ image, and used to make color images $(B-I$ and $V-I)$. On both color images, next to the red east-west feature, we also detected the blue region on the minor axis. These tests suggest the color features on the last panel of the Fig. 2 are real, although could be augmented by the convolution process due to its proximity to the saturated nucleus.

The remaining and real (clearly visible on all test images), thin blue feature follows the major axis of the galaxy, along which there is clear evidence for a very thin disk on all scales, from the nucleus outwards. The position angle of the thin blue component is the same as of the nuclear stellar disk.

The marginal difference in color $(\approx 0.01-0.02 \mathrm{mag})$ is almost razor sharp and it looks like the signature of the thin disk visible also in the residual image (second row of Fig. 2). We compared the sizes of the disk in the residual image and the blue feature in the $B-I$ color image. The comparison is made by extracting and averaging together several profiles of intensity and color perpendicular to the disk, on both sides of the nucleus (avoiding the central $2^{\prime \prime}$ ). The final profiles were fitted with Gaussians. The size $(F W H M)$ of the disk feature on the residual disk image is $\sim 5.6$ pixels and the size of the color feature is $\sim 4.4$ pixels. These numbers correspond to 0.25 and 0.20 respectively, and they are in good agreement, enforcing the connection between the components. At the distance of NGC 5308, the blue component in the color image is approximately $30 \mathrm{pc}$ thick.

A way to quantify the relative difference in color between the disk and the bulge is shown in Fig. 3. We measured the color along slit-like apertures ( 1 pixel wide) along the disk and parallel to it, on both sides of the nucleus. The central $1^{\prime \prime}$ of all slits were omitted, and the two slits, positioned on each side of the central slit, were averaged and presented as one color profile. The disk is clearly bluer then the bulge in the inner $8^{\prime \prime}$. Beyond 3.'5 on each side of the center colors of the disk and bulge become similar.

Briefly summarizing, we list below the important observed color features to which we refer later in the text:

NGC 4128 has (i) a red nucleus, and (ii) a blue feature 0 !' 14 west and 0 .'32 north of the galaxy center;

NGC 4621 has (i) a blue nucleus, (ii) an extended blue component with $\mathrm{PA} \approx 150^{\circ}$ (east of north), and (iii) an extended red component with $\mathrm{PA} \approx 268^{\circ}$ (east of north);

NGC 5308 has (i) an extended blue component along the major axis, and (ii) a wide blue component along the minor axis, (iii) an extended red component with an east-west orientation.

\section{STIS spectroscopy}

Spectra of all four galaxies were obtained using the Space Telescope Imaging Spectrograph (STIS) with aperture 52" $\times$ 0.2 and grating G430M (for details see Kimble et al. 1998). The observations are summarized in Table 5. The configuration of STIS and the properties of the grating are listed in Table 6. With this setup we chose to observe $\mathrm{Mg}$ lines at $5180 \AA$ rather then $\mathrm{Ca}$ lines at $8700 \AA$ which are commonly used for extracting stellar kinematics. The reasons were: (i) the $\mathrm{Mg}$ lines provide simultaneous kinematic data and a commonly used index of stellar metallicity; (ii) STIS shows problems with scattered light inside the CCD chip in the near infra-red which generates artificial "wings" on spectral features; and (iii) the spatial resolution along the slit in $V$-band is better by almost a factor of two due to the decreased Airy disk size.

\subsection{Data reduction}

The galaxies were observed in a similar manner with a total of four orbits per galaxy. Spectra were taken at three parallel 
positions per galaxy during the four orbits. Each orbit was divided (CR-SPLIT) into 3 shorter exposures. Two orbits were used for the slit placed on the center of the galaxies (cen), along the major axis. Between orbits, the galaxy was shifted along the slit for about 0.2 , or 4 pixels, to get a better estimate of detector sensitivity variations and to identify hot pixels. This strategy was not successful for the case of NGC 4128, where the measured shift was $\sim 1$ pixel. The remaining two orbits were split between two slit positions on either side of the central slit, covering the bulge parallel to the nuclear stellar disk. One slit was targeted at the position +0. ' 3 away from the central slit (positive offset - pos), and the other at the position -0.3 away from the central slit (negative offset - neg).

Most of the data reduction was performed by the HST/STIS calibration pipeline CALSTIS (Hodge et al. 1998), including subtraction of overscan, bias and dark, then flat-fielding, hot pixel and cosmic-ray removal, absolute sensitivity calibration and wavelength calibration. CR-SPLIT data sets were combined automatically in the pipeline. The additional combination of the two central slit exposures was performed manually outside the pipeline in IRAF, using the STSDAS task MSCOMBINE. This task averages the exposures scaled by their exposure times, and combines the separate exposures using a robust sigma clipping rejection method. This was done on the files that have _crj extension, i.e., after pipeline coaddition of CR-SPLIT images and before the calibrations. The combined files were then returned to CALSTIS for the calibrations. The same procedure, however, was not possible on the side slits which were taken during only one orbit. As there were still some cosmic rays left after the pipeline reduction, we used Laplacian Cosmic Ray Identification (LAcosmic) developed by van Dokkum (2001) to remove them. LAcosmic was also applied on the _crj files. The detection limit for the outliers was $3.5 \sigma$. To improve the quality of the images and remove additional negative pixels we tried a few techniques. For spectra with very low signal-to-noise it was possible to compare different exposures (e.g. the two side slits) and to recognize the same negative pixels and create a mask of them. For spectra with higher signal to noise this was not effective and a different approach was used. Using a boxcar filter we smoothed each LAcosmic-filtered image. These images were subtracted from the corresponding original LAcosmic-filtered images to emphasizes the outlying pixels. They were flagged creating a mask image. Masked pixels were interpolated using IRAF task FIXPIX. The resulting images were returned to CALSTIS and processed to the end of the pipeline.

The final STIS light profiles are shown in Fig. 4. A noticeable feature is the difference in the intensity of the side slits. If the centering and shifting process worked properly, as the light profiles of the galaxies are quite symmetric, it is expected that the side slits should have very similar profiles. This is true for the case of NGC 5308 and NGC 4621, suggesting those slits were on similar but opposite positions. However, the other two galaxies show significant deviations. It is therefore necessary to find the exact positions of all slits.

We checked the actual positions of the slits by comparing the light profiles from the STIS spectra with the WFPC2 images. The width of the slit (dispersion direction) is 0.2 , which corresponds to 4 pixels on the STIS CCD. We sub-sampled the image such that the slit width projects to 5 pixels, in order to center the slits more correctly. Summing up along the dispersion axis ( $x$-axis on the CCD) we created a STIS light profile. This was repeated for all slits. The $F 555 \mathrm{~W}$ images, which were used in the comparison, were accordingly re-sampled. In each case we scanned the WFPC2 image by a combination of the three slits, independently varying the distances between the slits. The comparison of the STIS and WFPC2 profile was expressed by the relative $\chi^{2}$ (profile $(W F P C 2) /$ profile $\left.(S T I S)-1\right)$. In this process we assumed that the position angle of the telescope did not change between different slit positions. The resulting $\chi^{2}$ estimates are shown in Fig. 5. If the central slit is not on the nucleus, the $\chi^{2}$ is expected to have double minima and it is hard to distinguish which one is correct (NGC 4621 and NGC 4570 are clear examples). However, using the additional light profiles of the side slits tightens the constraints producing one clear minimum, which corresponds to the position of the central slit. The uncertainty of our estimate is $0 .^{\prime} 04$. The positions of the central and the side slits with respect to the nucleus are given in last two columns of Table 5. The slits in NGC 5308 and NGC 4128 are centered on the galaxy nucleus, while for NGC 4621 and NGC 4570 the slits were offset. The side slits were roughly on the requested positions for NGC 4621 and NGC 5308. In the case of NGC 4128, the side slits are the least symmetrically positioned and the pos slit is almost coincident with the central slit; while in the case of the NGC 4570 the positions of the side slits are the farthest apart, as suggested from the profiles (Fig. 4).

The general characteristic of the spectroscopic data is their low signal-to-noise ratio $(S / N)$. Only the major axis spectra have an $S / N$ sufficient for the extraction of kinematics and linestrengths as a function of radius. The side slits are much noisier and no kinematic measurements were possible. It was possible however to extract line-strength information from a few central rows, summed together to increase $S / N$ creating one spectrum per side slit per galaxy.

\subsection{Stellar kinematics}

All available information about the stellar kinematic properties of galaxies are given by the line-of-sight velocity distribution (LOSVD). The process of extracting kinematics is based on the deconvolution of the observed galaxy spectra in order to recover the full LOSVD. The idea behind this is that the galaxy spectrum can be reproduced using a combination of several representative stellar spectra convolved with the true LOSVD. Unfortunately, the LOSVD is not a priori known and the deconvolution process is ill-determined, being heavily dependent on the quality of the data. Over the last thirty years a number of methods were invented to tackle the problem and deliver the best possible estimates of the LOSVD (see de Bruyne et al. 2003, for an overview of methods). Here we choose to use a parametric method operating in the pixel space because of the low $S / N$ of our data and very short wavelength range. We use the penalized pixel fitting (pPXF) method (Cappellari $\&$ Emsellem 2004). We derive the LOSVD parameterized by a 
Gauss-Hermite series (van der Marel \& Franx 1993; Gerhard 1993). The method finds the best fit to a galaxy spectrum by convolving an optimal template spectrum with the corresponding LOSVD given by the mean velocity $V$ and velocity dispersion $\sigma$, as well as higher order Gauss-Hermite moments $h_{3}$ and $h_{4}$. The higher order moments measure asymmetric and symmetric deviation of the LOSVD from a Gaussian respectively.

An element which can heavily influence the extracted kinematics is the stellar template used to convolve the LOSVD to reproduce the galaxy spectra. There are methods, such as Fourier correlation quotient (Bender 1990) or Cross-correlation method (Statler 1995), which are less sensitive to template mismatch. Pixel fitting techniques are much more sensitive to template mismatch and it is crucial to have a good stellar template before starting the extraction. The usual way is to observe a number of representative stars (matching the spread in metallicity and age of stars in the observed galaxy) with the same instrumental set-up and to build an optimal template as a weighted linear combination of the observed stellar spectra.

After searching through the HST archive we decided to use a set of stellar population models instead of the one star from the archive that matched our set-up (the same grism being the most important, while size of the slit can be accounted for). Using single-metallicity stellar population models of Vazdekis (1999) we constructed a large stellar library from which to build the optimal stellar template. Each galaxy long-slit spectrum was summed up along the slit to make a higher $S / N$ spectrum, which was used to obtain the optimal template. We also used additive Legendre polynomials to adapt the continuum shape of the templates. This optimal template was then used in the fit of the individual spectra along the slit.

A disadvantage of the Vazdekis models is that they are of lower resolution than the STIS data. The FWHM of the Vazdekis library is $1.8 \AA$ compared to $0.8 \AA$ from STIS. This requires a degradation of our data by $\sim 1.6 \AA$. Although certain information is in this way lost and the STIS spectral resolution is degraded, the smoothing of the data helps in removing noise and the library ensures the extracted kinematics do not suffer from an important systematic template mismatch effect. Examples of constructed optimal templates are shown in Fig. 6. The presented spectra are the sum of the central 10 rows, having a high $S / N$ which is needed for properly estimating the optimal template. The overplotted dashed lines are our resulting optimal templates for the galaxies, convolved with the determined LOSVD of the galaxy. Typically a few (2-3) old-type stars from the Vazdekis library were selected by the fitting routine for the optimal template. The residuals between the galaxy and the optimal template spectra are shown below each spectrum. The presented optimal templates were used to extract the kinematics from the spatially binned spectra. An alternative way would be to construct the optimal template for each spatial bin and then use this to extract kinematics in the same bin. This method is important for galaxies with stellar populations changing between bins ( 0.'05), but in this case the low $S / N$ of the individual spectra do not justify this approach.

Table 7 summarizes the details about the spatial bins used for the extraction of kinematics. They were chosen after some

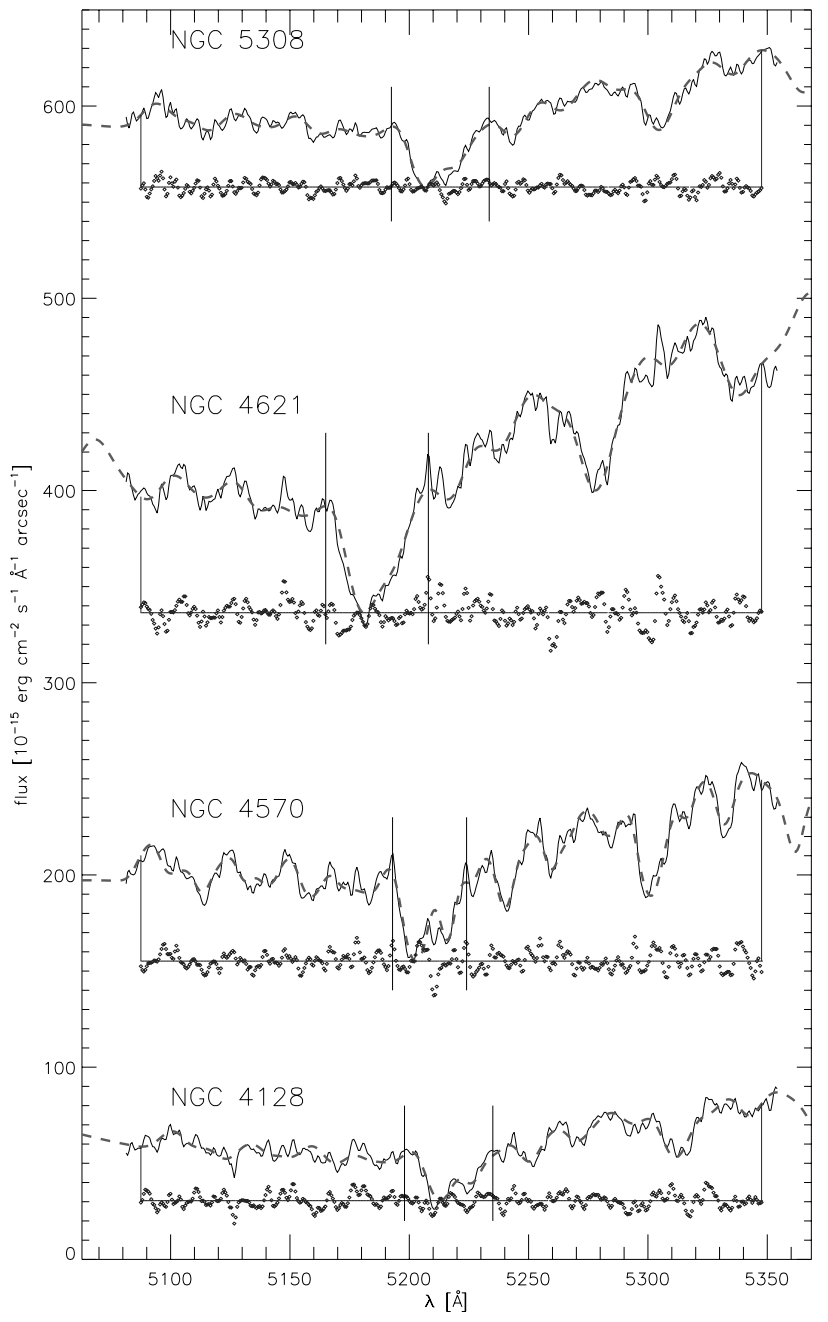

Fig. 6. Example of spectra for the four studied galaxies. From bottom up: NGC 4128, NGC 4570, NGC 4621 and NGC 5308. The spectra are shifted vertically to avoid overlap. The thin black lines indicate spectra of galaxies. The dashed thick lines are broadened optimal templates. The dots below the spectra are residuals of the fit (difference between galaxy spectra and optimal templates). Vertical and horizontal lines shows the region used in the fit. Two solid vertical lines crossing the spectra show the spectral regions excluded from the fit which tested the influence of $\mathrm{Mgb}$ region to the extracted kinematics.

experimenting as a compromise between the $S / N$ and the spatial resolution. The galaxies have different surface brightnesses and, since the exposure times were similar, a unique scheme for all galaxies was not useful. For each galaxy we assumed a target $S / N$ and we binned accordingly. Generally, the spectra become too noisy to measure the kinematics beyond $1^{\prime \prime}$. In some cases, the central few rows of spectra have $S / N$ high enough for extraction of the higher order terms of LOSVD, but in general the $S / N$ is too low. Hence, we decide to confine the extraction to only the first two moments (assuming a Gaussian shape for LOSVD): mean stellar velocity $(V)$ and velocity dispersion $(\sigma)$.

Another element which can heavily influence the results of the extraction is specific to the spectral region of the observations. Barth et al. (2002) compared the kinematics of a number of galaxies extracted in two spectral regions: one around 
$\mathrm{Mg} b$ lines and the other around Ca triplet. They found that if the metallicities of the galaxies and template stars are not well matched then direct template-fitting results are improved if the $\mathrm{Mg} b$ lines themselves are excluded from the fit and the velocity dispersion is determined from the surrounding weaker lines. For galaxies with high velocity dispersion this will be more important because of the correlation between the velocity dispersion and the $[\mathrm{Mg} / \mathrm{Fe}]$ ratio (Worthey et al. 1992; Trager et al. 1998; Kuntschner et al. 2001), which increases the strength of the $\mathrm{Mg} b$ lines relative to the surrounding Fe lines. Following the suggestion of Barth et al. (2002) we also extracted kinematics excluding from the fit the $\mathrm{Mg} b$ lines (the excluded regions are shown in Fig. 6 as vertical lines crossing the spectra). When there are significant differences between the two sets of extracted kinematics we used the set obtained by excluding the $\mathrm{Mg} b$ lines from the fit for the further analysis and interpretations.

The errors were estimated using Monte-Carlo simulations. The LOSVD parameters were derived from 100 realizations of the input spectrum, where the value at each pixel is taken from a Gaussian distribution with the mean of the initial spectrum and standard deviation given by a robust sigma estimate of the residual of the fit to the initial spectrum. Figure 6 shows an example of the residuals used to estimate the standard deviation used in Monte-Carlo simulations (dots under the spectra). All realizations provide a distribution of values from which we estimate the $1 \sigma$ confidence limits. The values of the extracted kinematics are presented in Tables A.1 and A.2 of Appendix A and shown in Figs. 8-11.

All galaxies except NGC 4621 show rather fast major axis rotation. NGC 4621 is a special case with a previously discovered counter-rotation in the center (WEC02). There are some differences between the kinematics extracted fitting the full spectral range and excluding the $\mathrm{Mg} b$ lines. They are the strongest for NGC 4621 and NGC 4570. The somewhat larger error bars of the kinematic measurements obtained not fitting the $\mathrm{Mg} b$ region are the consequence of lowering the $S / N$ by excluding the dominant spectral feature. We postpone detailed description of all kinematic curves to Sect. 4.

\subsection{Line strengths}

The spectral range of our observations is very limited covering only the $\mathrm{Mg} b$ and Fe5270 Lick/IDS indices (for definition of Lick/IDS system and indices see Burstein et al. 1984; Worthey et al. 1994; Trager et al. 1998). The red continuum pass band of the Fe5270 index is truncated by the edge of our spectral range and this index cannot be used in its defined form. A similar case is found in Kuntschner et al. 2004 (in preparation) and Falcón-Barroso et al. (2004) where Fe5270 cannot be mapped over the whole field-of-view of the integral-field spectrograph SAURON (Bacon et al. 2001) due to the varying bandpass of the SAURON instrument. In their case, Kuntschner et al. (2004) redefine the index to maximize the coverage of the field-of-view and retain the sensitivity of the index towards changes in age, metallicity and abundance ratios. The new index name is Fe5270s. It measures the same spectral feature, but has a reduced spectral coverage in the red pseudocontinuum band. The new index can be converted to the original Lick/IDS system via the empirical formula (Kuntschner et al. 2004; Falcón-Barroso et al. 2004):

$\mathrm{Fe} 5270=1.26 \times \mathrm{Fe} 5270 s+0.12$.

The $1 \sigma$ standard deviation of the above empirical calibration is $\pm 0.05 \AA$ for the Fe5270 index. More details on the derivation of the new index and its relation to the standard Lick/IDS index are given in Kuntschner et al. (2004).

Having this in mind we measured Fe5270s and $\mathrm{Mg} b$ indices. The Mg $b$ index was measured using the Lick/IDS index definition, and all spectra were first broadened to the resolution of the Lick/IDS system. The Fe5270s index was later converted to index Fe5270 using above relation. Unfortunately, we were not able to determine the relevant offset to the Lick/IDS system, and correct for the systematics, which come from differences in the continuum shape, because there are no reference stars in the HST archive observed by our and by the Lick/IDS instrumental setup. The size of the corrections are probably similar to (or less than) our measurement errors. To first order, as well as for determining the relative trends in a galaxy, this is not very important, but has to be noted when comparing with other studies.

Broadening of the lines by the velocity dispersion weakens most of the lines and the index we measure must be corrected for this effect. This can be achieved by determining an empirical correction factor $C(\sigma)=\operatorname{index}(0) / \operatorname{index}(\sigma)$ for a star observed with the same instrumental setup. $\operatorname{Index}(0)$ is the index measured from the stellar spectrum, $\sigma$ is the velocity dispersion of the LOSVD with which the stellar spectrum is convolved and from which the index $(\sigma)$ is measured. We used our unbroadened optimal template spectra to calculate the index at $\sigma=0$ and at the corresponding velocity dispersion, $\sigma$, of the galaxy spectrum. The proper correction factor $C(\sigma)$ was then applied to both measured indices. We used two approaches to extract kinematics and measure the velocity dispersions (fitting the whole spectral region and excluding $\mathrm{Mg} b$ region from the fit). If the measured velocity dispersions differ, the velocity dispersion correction in the two cases will also be different. We noted the difference applying both corrections on the measured line-strengths.

We measured the $\mathrm{Mg} b$ and Fe5270 indices from each spectral bin used for kinematics. The corrected values and corresponding errors of the index are presented in the Tables A.1 and A.2 and shown in the Figs. 8-11. The measured linestrengths for galaxies with higher $S / N$ are relatively uniform with radius, rising towards the center, with dips in the case of the nuclei of NGC 4621 ( $\mathrm{Mg} b$ ) and NGC 5308 (Fe5720). NGC 4128 does not show any trend, but rather a scatter of values, presumably due to the low $S / N$, while in case of NGC $5308 \mathrm{Mg} b$ line-strengths are slightly higher on one side of the galaxy. Generally, galaxies have high values of $\mathrm{Mg} b$ and Fe5270 indices. Detailed descriptions of spatially resolved line-strengths for all galaxies are given in Sect. 4.

The slits that were offset to the sides of the central slit do not have the required $S / N$ to extract kinematics, but their summed spectra can be used to determine the indices on the positions of the galaxy outside the stellar disk. The binning of 
spectra is only useful up to the point at which summing more spatial elements does not simply add noise. We decided to use an aperture of $0.55 \times 0 . ' 2$ (summing up 5 rows on each side of the central row in the spectral direction). With this approach the final side spectra used for the measurements of line-strengths had at least $S / N \approx 10$. In the case of NGC 4128, however, from one side spectra we were not able to extract any trustworthy measurement. Table 8 presents line-strengths corrected for velocity dispersions measured by fitting to the whole spectral range and excluding the $\mathrm{Mg} b$ lines from the fit. As it can be seen, the differences between the lines are negligible and generally fall within the $1 \sigma$ error bars. Only in the case of the central slit of NGC 4621 which has the highest velocity dispersion as well as $S / N$, there is a significant difference. Adopting a conservative approach, we compared line-strengths from the second two rows in Table 8 with the stellar population synthesis models in Fig. 7.

We wish to compare the line-strengths measured on the disk with the line-strengths measured on the bulge using the three slit positions. The line-strength measurements on the summed spectra show similarly high values as the spatially resolved measurements, although with relatively lower values due to the smaller spatial resolution. The Mgb index values in NGC 4621 are particularly high. Comparing with the literature we find similar values for $\mathrm{Mg} b$ and Fe 5270 index. Table 7 of Trager et al. (1998) list values of the same indices for NGC 4621 and NGC 4570 (Mgb 5.50 and $4.65 \AA$, Fe5270 3.59 and $3.49 \AA)$, which, keeping in mind the unknown offset to the Lick system and the lower spatial resolution of Trager et al. (1998) data (aperture of $1^{\prime \prime} \times 4^{\prime \prime}$ ), are in good agreement with our findings.

Age and metallicity have similar effects on the integrated spectral energy distributions that we measure from unresolved sources due to a finely tuned conspiracy between age and metallicity variations (Worthey 1994). Broad-band colors and many line-strength indices are degenerate with respect to age and metallicity. This makes the determination of the age and metallicities very difficult and ideally one would like to use two indices which can break this degeneracy. Usually, one or more Balmer lines $(\mathrm{H} \beta, \mathrm{H} \gamma, \mathrm{H} \delta)$ are used as age indicators, and $\mathrm{Mg} b$ or some Fe index (Fe5270, Fe5335) as a metallicity indicator (González 1993; Fisher et al. 1996; Mehlert et al. 1998; Kuntschner 2000; Trager et al. 2000). The high index values of our measured line-strengths also suggests the presence of non-solar abundances of elements. If not properly treated, over-abundant indices can give wrong age and metallicity estimates (Kuntschner et al. 2001). A way around this issue is to define metallicity indicators which are insensitive to abundance ratios (González 1993; Thomas et al. 2003). The preferred indicator includes a combination of $\mathrm{Mg} b, \mathrm{Fe} 5335$ and Fe5270 indices, where Fe5270 is the least sensitive to changes of $[\alpha / \mathrm{Fe}]$ abundance ratios (Thomas et al. 2003). We were not able to construct such a metallicity indicator with the indices from our spectral range, and we chose to use the least sensitive Fe5270 index alone as a metallicity indicator. Since in our spectral range there are no age indicators, we decided to use a combination of broad-band $B-V$ colors and Fe5270 index to construct an age/metallicity diagnostic diagram (Fig. 7). The models presented by solid lines are based on the

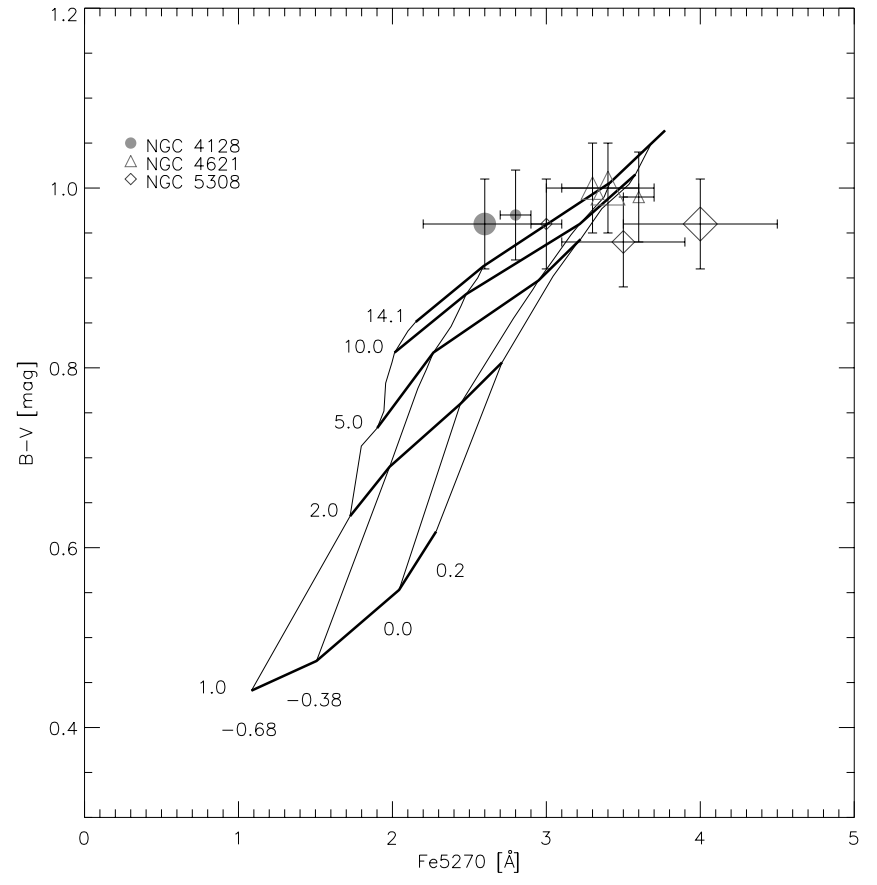

Fig. 7. Age/metallicity diagnostic diagram ( $B-V$ color vs. Fe5270 index). Horizontal thick solid lines are lines of constant age $[\mathrm{Gyr}]$ and vertical thin lines are lines of constant metallicity $[\mathrm{Fe} / \mathrm{H}]$ ) of Vazdekis (1999) models. The size of symbols is related to the position of the slit: the smallest symbols are for the cen slits, intermediate for the pos slits, and the biggest for the neg slits.

Vazdekis (1999) single stellar population models: color values were obtained from A. Vazdekis web site ${ }^{3}$, while we measured the Fe5270 index from the library spectra broadening them to the Lick/IDS resolution. The combination of red colors and high metallicities puts the measured points in Fig. 7 on the top right of the model grid, indicating old stellar populations and a large spread of metallicities between the galaxies.

\section{Discussion}

In the two previous sections we presented the observational results of HST program 8667. They include photometric and spectroscopic observations of four galaxies with nuclear stellar disks. Here we analyze and discuss the observations.

\subsection{NGC 4128}

The most distant galaxy in the sample is NGC 4128 (36 Mpc). It is an S0 galaxy and it has not been detected in radio nor in IR. The isophotal parameters show that it is disky between 35 and $530 \mathrm{pc}$. On $1 \mathrm{kpc}$ scale it has boxy parameters and on larger scales it becomes disky again.

The color image shows a red nucleus. The values for Fe5270 index measured with an aperture bigger than the red nucleus are the smallest in the sample of galaxies. This combination puts the points in Fig. 7 above the model grid.

\footnotetext{
${ }^{3}$ http://www.iac.es/galeria/vazdekis/
} 
The difference in colors and line-strengths between the two slit positions are small and within errors indicate old stellar populations of $\sim 14 \mathrm{Gyr}$ and metallicities between $[\mathrm{Fe} / \mathrm{H}]=-0.38$ and solar.

It is probable that the difference in the color between the nucleus and the rest of the galaxy, as well as the higher metallicity detected in the nucleus is connected with the unusual spatially resolved kinematic profiles (Fig. 8). The velocity dispersion is flat in the center. The velocity curve also shows an unusual flattening in the central 0.2 , measurements being positive on the both sides of the galaxy nucleus. Outside this radii the galaxy rotates fast, as expected for a disk galaxy. Kinematics extracted fitting to two different spectral regions are in a good agreement, confirming the results. Having in mind the boxiness in the central tens of arcseconds, the extracted kinematic indicates the existence of a small ( $\sim 35 \mathrm{pc}$ in diameter) core, kinematically distinct from the nuclear stellar disk.

The $B-V$ color profile on the last panel of Fig. 8 shows a slightly shifted $(\sim 17 \mathrm{pc}$ from the center) peak of the red nucleus. This supports the presence of a distinct component in the nucleus. On the other hand, the spatially resolved linestrengths do not follow this trend. The spectral observations of this galaxy have the smallest $S / N$ ratio, and the significance of this discovery is just above $1 \sigma$. Deeper exposures of high spatial resolution, preferably with an integral-field unit to cover the 2D structure, are needed to confirm this result.

\subsection{NGC 4570}

NGC 4570 is a well-studied galaxy with HST. The main result from previous studies is that the inner region of the galaxy was shaped under the influence of a weak bar (van den Bosch \& Emsellem 1998). The colors reveal no difference between the disk and the bulge, and a comparison with the stellarpopulation models indicate that the stars in the galaxy are of intermediate age, but the FOS spectral data gave a very high $\mathrm{H} \beta$ line-strength suggesting recent star formation (BJM98). One of the questions raised by these studies is whether all double-disk structures are the result of bar-driven secular evolution.

To the previous photometric and spectroscopic observations we add new spatially resolved spectroscopic measurements with STIS (Fig. 9). The probed region corresponds to the nuclear disk and inner $2^{\prime \prime}$. The velocity curve shows regular rotation peaking at $\sim 0$.' 15 from the nucleus. The velocity dispersion steeply rises and peaks in the center. The kinematic profiles are similar to BJM98 ground based data, except the STIS data have a steeper velocity curve and higher velocity dispersion. In contrast, the FOS velocity dispersion from the same authors is about $50 \mathrm{~km} \mathrm{~s}^{-1}$ higher than STIS measurements; however, considering the error bars of both measurement (their error on sigma is $\approx 30 \mathrm{~km} \mathrm{~s}^{-1}$ ) and the fact that our slit was significantly (for the width of the slit) offset from the galaxy nucleus, these measurements can be considered consistent with each other.

In the case of NGC 4570, the central velocity dispersion is somewhat dependent on the spectral region used in the fit.
Excluding $\mathrm{Mg} b$ region systematically lowers the values by just over $1 \sigma$, but increases the difference between this and BJM98 results.

We also measure, within the errors, similar line-strengths to BMJ98, but with higher spatial resolution and we can give an estimate of the spatial changes in the indices. As can be seen from the Fig. 9, both measured indices show flattening in the central 0.5 ( $\sim 60 \mathrm{pc})$. At larger radii the metallicity drops. Also, the slits positioned on both side of the center measure the smallest metallicity (Table 8) in the sample and the largest drop in values with respect to the center. This measurement shows that the nuclear disk consists of different stellar populations than the rest of the bulge, which is consistent with bar-driven evolution.

\subsection{NGC 4621}

The closest galaxy of the four is NGC 4621 (7 Mpc). It is also the only galaxy classified as an elliptical and is the only galaxy from the sample detected with IRAS (in the $12 \mu \mathrm{m}$ band). In the investigated range the galaxy is disky. The $b_{4}$ coefficient steadily rises from the center to the distance of $140 \mathrm{pc}$ when it drops, but never reaching negative values. With increasing radius it rises again, implying an outer disk.

The color images reveal the most interesting features in the nucleus. The few central pixels are clearly much bluer than the rest of the bulge (Table 4). Another striking characteristic of the galaxy, mentioned in Sect. 2.3.2, is the extended blue and red features visible on $B-I$ (Fig. 2) and $V-I$ images. The blue feature makes an angle of $15^{\circ}$ with the major axis. Although the red feature spreads generally in the east-west direction (angle with major axis is $103^{\circ}$ ), it is not as clearly defined as the blue feature. We conclude, examining all color images, that the shape and the extent of the two features can be interpreted as a blue disk-like structure imbeded in the red bulge. The position angle of the blue feature is unexpected for an axisymmetric galaxy with a nuclear stellar disk. This significant structure perhaps can be explained considering the kinematics of this galaxy.

The velocity and velocity dispersion panels in Fig. 10 clearly show the existence of a kinematically decoupled core (KDC). This core was already detected by WEC02 who observed the galaxy with integral field spectrograph OASIS mounted on CFHT and assisted by the PUEO adaptive optics system. Complementing their OASIS observations, WEC02 also extracted kinematics from an archival STIS observations in the $\mathrm{Ca}$ triplet region, showing a peak in velocity dispersion 0 .'05 from the center as well as confirming the KDC with the same spatial resolution as in the data presented here. The difference between the WEC02 and our kinematic profiles is largest in the velocity dispersion profile, where our values lie systematically above the WEC02 measurements. This difference is lower, but still present if we compare the kinematics extracted excluding the $\mathrm{Mg} b$ line from the fit $\left(\sim 60 \mathrm{~km} \mathrm{~s}^{-1}\right.$ for the velocity dispersion peak, but with large error-bars in both cases). This discrepancy could arise from the different slit positions in the two studies, our slit being offset from the center and not covering the KDC uniformly. The counter-rotation of 
the KDC could lower the overall measured dispersion if the slit is placed over its center, and, alternatively, if the slit misses the center of the KDC the measured velocity dispersion will be higher.

The KDC on the WEC02 OASIS data is not aligned with the major axis and it has a similar position angle as the blue feature on the color images presented here. Although the extent of the KDC is smaller (total of $\sim 2^{\prime \prime}$ or $60 \mathrm{pc}$ ) than the blue feature on the $B-I$ image, the existence of two structures could be connected as a result of the same formation process.

Both $\mathrm{Mg} b$ and Fe5270 line-strengths indices in NGC 4621 are the highest in the sample, also suggesting the overabundance ratios of elements similar to trends for giant ellipticals (Kuntschner 1998; Kuntschner et al. 2001). Our metallicity indicator, Fe5270, also has high values, with the central slit being slightly more metal-rich than the side slits as well as super-solar. Colors at the slit positions are red and the comparison with the stellar population models indicates the age of the stars is between 10 and $14 \mathrm{Gyr}$ and the metallicity between solar and +0.2 .

Our spatially resolved measurements of the indices, shown in Fig. 10, are higher than in previous studies (e.g. Kuntschner et al. 2001, have central $\operatorname{Mg} b \sim 5.21$ with aperture of 3.' 4), but our aperture is much smaller $\left.(\sim 0)^{\prime} 05\right)$ than that of any previous study, and the values outside the central arc-second approach the observed values from the literature. The $\mathrm{Mg} b$ index follows to some extent the changes in colors, showing a small dip in the center, while this can not be said for Fe5270 measurements.

The existence of the KDC and the blue features in the red bulge of the NGC 4621 indicate two possible evolutionary scenarios. The visible structures could be the result of a hierarchical formation scheme (e.g. Kauffmann et al. 1994) involving a merger followed by a starburst where the KDC is the remnant of the ejected stars that later fell back in. These structures are relatively long lived, having a relaxation time of $\sim 1 \mathrm{Gyr}$ (Binney \& Tremaine 1987); however, this is not long enough to explain the detected old age of the stars. Alternatively, the structure could be produced by weak bar-driven evolution, as in the case of NGC 4570 (van den Bosch \& Emsellem 1998), where the observed double disk structure is the consequence of resonant frequencies in the galaxy, while the blue feature and the KDC are the result of gas captured on retrograde ("anomalous") orbits which are tilted with respect to the equatorial plane (Pfenniger \& Friedli 1991; Friedli \& Edry 1993; Emsellem \& Arsenault 1997). Of course, a combination of both processes can also lead to the present situation.

Distinguishing between the two scenarios is also difficult because the galaxy has no obvious merger companion and it is nearly edge-on, making the detection of a weak bar more difficult. There are other cases of barred galaxies with similar properties to NGC 4621: i.e. an edge-on system with doubledisk structure, unusual photometric and kinematic features and the difference in metallicity between the bulge and the disk. An example of a similar, although boxier, galaxy with a strong bar is NGC 7332. This galaxy was recently studied in detail by Falcón-Barroso et al. (2004). NGC 7332 is classified as an S0 galaxy and has a double disk structure (Seifert \& Scorza 1996). Examining their SAURON spectroscopic observations,
Falcón-Barroso et al. find a counter rotating stellar component within the central $250 \mathrm{pc}$. The galaxy also has complex gas morphology and the line-strength maps show it is young everywhere. The authors conclude that NGC 7332 is an S0 galaxy with a bar viewed close to edge on. NGC 4621 and NGC 7332 are similar in their morphologies, and, although different in the stellar content, it is possible that NGC 4621 went through a similar formation process as NGC 7332.

\subsection{NGC 5308}

In many aspects NGC 5308 is different from the other galaxies in this study. Our photometry reveals the largest nuclear stellar disk in the sample of galaxies in this study. Unlike in the other galaxies, the nuclear disk of NGC 5308 is very thin and bright. The diskiness parameter, $b_{4}$, rises from the center and peaks at about $150 \mathrm{pc}$, dropping to zero at $\sim 1 \mathrm{kpc}$ and suggesting a distinction between the two disks. At large radii the galaxy again becomes disky.

The stars in the disk of NGC 5308 rotate fast, reaching $\sim 100 \mathrm{~km} \mathrm{~s}^{-1}$ within $15 \mathrm{pc}$ from the nucleus (Fig. 11). The velocity dispersion has a peak of about $300 \mathrm{~km} \mathrm{~s}^{-1}$ in the center and is relatively flat in the inner $15 \mathrm{pc}$. This trend is also visible in the kinematics measured excluding the $\mathrm{Mg} b$ line from the fit, although the right hand side of the plot shows considerably lower velocity dispersion values. This is reflected in the panel with $\mathrm{Mg} b$ values, which are slightly higher on the right hand of the plot. The $\mathrm{Mg} b$ and Fe5270 index values have opposite trends. The small variations in the $B-V$ color along the slit, including the sudden blue dip in the center, are followed by the line-strength measurements.

There is a big difference between the line-strengths measured on the different slit positions (Table 8 and Fig. 7). The nucleus, being just below solar metallicity, is more metal-poor than the bulge which has a non-solar abundance ratio of elements and the highest metallicity in the sample. The nucleus and the investigated part of the bulge also have different colors, with the center being redder. Comparing these results with the stellar population synthesis models reveals an old stellar population in the nucleus (14 Gyr), while the colors of the bulge suggest intermediate age stellar component ( 5-10 Gyr).

The color difference between the bulge and the nucleus is measured because the side slits were positioned in the region of the minor axis blue feature, especially visible on $B-I$ image. This region is also special for its high metal content. There are no hints of specific morphological structures (such as a polar ring) along the minor axis and the question is whether the rest of the bulge, especially the red feature, and the nuclear stellar disk share the same metallicity. Unfortunately our spectral observations did not cover the necessary areas and we can only speculate on the processes that created the observed structures.

If the metallicity of the minor axis blue feature and the nuclear disk are the same, the formation of these two structures has to be connected to the same formation scenario, most probably involving a transportation of gas to the center, perhaps by a bar, for which Seifert \& Scorza (1996) found evidence in NGC 5308. If the metallicity of the nuclear disk is lower than 
the metallicity of the minor axis blue feature, but similar to the measured metallicity of the nucleus, the two blue features in NGC 5308 were not created from the same infalling material, but they still can be from the same epoch. If the metallicity of the minor axis blue feature is equal to the rest of the bulge, which is redder and therefore older, then the younger stellar population in the blue feature must have been induced by an internal process, perhaps ionization from the radiation generated by the central black hole, which was turned on with the infall of the material that made the blue disk, and later turned off with the stabilization of the disk (Loeb \& Rasio 1994).

In Sect. 2.3.2 we showed that the major axis blue feature in NGC 5308 corresponds to the nuclear stellar disk. It is about $30 \mathrm{pc}$ thick which strongly suggest the galaxy is viewed very close to edge-on. Comparing with the vertical scalelength of 34 edge-on spirals presented by Kregel et al. (2002), which are between 0.2 and $1.4 \mathrm{kpc}$ thick, the disk in NGC 5308 is a remarkably thin disk. Note that our estimate of the disk thickness can only be approximately compared with the vertical scalelength measurements of Kregel et al. It is also not possible to say much about the thickness of the other nuclear disks, due to their inclination (not as edge-on as NGC 5308). Whether this nuclear disk resembles the disks from the group of "super-thin" galaxies, like UGC 7321 or IC 5249 (Matthews et al. 1999; Matthews 2000; van der Kruit et al. 2001), is an open question. The sizes of the nuclear disk in NGC 5308 and known "super-thin" disks are quite different as well as the surrounding environment (stellar bulges and dark matter halos respectively). A proper way to compare the disks is to measure the radial and vertical sizes in a consistent manner, which is beyond the scope of this paper.

The color and metallicity of the disk in NGC 5308 suggest the disk could be made of a younger and more metal-poor stellar population than the rest of the galaxy, implying it formed at a different epoch from accreted material.

\section{Conclusions}

We have presented photometric and spectroscopic observations of four nearby early-type galaxies with nuclear stellar disks (NGC 4128, NGC 4570, NGC 4621, NGC 5308). The observations consist of high resolution images with WFPC2 using the $F 450 W$ and $F 555 W$ filters, and STIS high resolution spectra through the $52 \times 0.2$ long-slit with the G430M prism.

The photometric analysis reveals similarities and differences between the galaxies. Nuclear stellar disks are clearly visible and are photometrically disconnected from the large scale disks. NGC 4128 shows boxy isophotes on the inner and outer edge of the nuclear disk. NGC 4621, the only E galaxy in the sample, is everywhere disky, while NGC 5308 has a razorthin $(\sim 30 \mathrm{pc})$ disk.

Color images reveal interesting and unexpected structures. NGC 4128 has a red nucleus, while NGC 4621 has a blue nucleus. Prominent color features are visible on all galaxies. The blue feature in NGC 4128 is analyzed in Appendix B and is the signature of a transient event. NGC 4621 has a blue feature at an angle of $15^{\circ}$ with the major axis on top of a red bulge. It is likely connected to the KDC discovered by WEC02.
The nuclear stellar disk in NGC 5308 is associated with the razor thin blue feature along the major axis. NGC 5308 has another blue feature along the minor axis. The colors of all three galaxies indicate old stellar populations except for the bulge of NGC 5308 where the combination of slightly less red colors and high metallicity lowers the age of the stellar populations.

The high resolution spectroscopy was obtained at three positions on each galaxy. One slit was positioned on the nuclear stellar disk, with the PA equal to the major axis PA. Two additional slits were positioned on both sides of the central slit, $\sim 0$ ' 3 away from the disk covering the bulge. The central slits were used to extract spatially resolved kinematics and linestrengths. The $S / N$ permitted the extraction of the mean stellar velocity and the velocity dispersion, as well as the measurement of line-strengths. The kinematics will be used in a separate paper to estimate the black hole masses in the centers of the galaxies.

Considering the shape of the spatially resolved kinematic curves, the four galaxies could be sorted in two groups: fast and kinematically disturbed rotators. NGC 4570 and NGC 5308 belong to the first group. Their rotation curves show clear signature of the stellar disks. The rotation curves of NGC 4128 and NGC 4621 are much more complicated. In the case of NGC 4621 the unusual mean velocity and velocity dispersion curves are consistent with the known KDC (WEC02) in the nucleus. Although based on a $1 \sigma$ detection, we report the discovery of a similar kinematically distinct core in the case of NGC 4128.

Spatially resolved line-strength measurements along the disk indicate that all four galaxies are more metal-rich in the inner 0.5 than outside this radius. Both measured indices $(\mathrm{Mg} b$ and Fe5270) increase towards the center, except in the case of NGC 5308 where Fe5270 has an opposite trend to $\mathrm{Mg} b$ index. Non-solar abundance ratios of $[\mathrm{Mg} / \mathrm{Fe}]$, hinted by results of the extraction of kinematics, are present in NGC 4570 and NGC 4621, and to some extent also in NGC 5308.

The slits positioned on the bulges had low $S / N$ and no spatially resolved kinematics were extracted. However, by binning the spectra, it was possible to measure the line-strengths at one position and compare them to the values of the nuclei. The objects show various structure: NGC 4128 has similar metallicities at the different slit positions, NGC 4570 and NGC 4621 have higher metallicity in the nucleus, while NGC 5308 in the bulge. Generally, the galaxies show a spread in metallicity from sub- to super-solar.

This study shows the diversity within this class of objects, but also emphasizes the similarities in the photometry and kinematics. The red color gradient in the nuclei of NGC 4128 and the blue features in NGC 4621 and NGC 5308 suggest the existence of different stellar populations on small scales ( 100-500 pc). The investigated galaxies were chosen as galaxies with specific nuclear morphologies: nuclear stellar disks. However, except in the case of NGC 5308 the colors of the disks are not much different from the bulge, as previously noted by Carollo et al. (1997b). The existence of other color features is a surprise. In two galaxies (NGC 4128 and NGC 4621) these color features are followed by the existence of a KDC. The other two galaxies do not show any 
peculiarities in their kinematics. Also, if the KDC in NGC 4621 is connected to the misaligned blue feature, we can conclude, similar to Carollo et al. (1997a), that KDCs are not kinematic counterparts of the nuclear stellar disks. This gives credit to the complexity of formation scenarios that demands a separate study per galaxy, but there are a few most likely frameworks, outlined also in Scorza \& van den Bosch (1998), in which the processes responsible for the observed structures operate.

The formation of nuclear disks, rings and double disk structures in early-type galaxies can be explained through secular evolution driven by weak bars as shown by Emsellem et al. (1996) and van den Bosch \& Emsellem (1998) in the cases of M 104 and NGC 4570, respectively. This mechanism, through the evolution of the bar, explains the double-disk morphology. Support for this scenario comes from the fact that S0 galaxies have high line-strengths (Fisher et al. 1996) and there are evidences of embedded bars in early-type galaxies (e.g. M 104, NGC 4570, NGC 7332, Scorza et al. 1998). This model of bardriven evolution is consistent with the observations in the presented galaxies, even in the cases of the galaxies with KDCs, such as NGC 4621, but also NGC 4128, which is additionally boxy and presents an interesting case. The time varying triaxial potential of the bars offers exotic orbits that could explain the existence of kinematic and photometric features. In this scenario, the KDCs are created from enriched material transported inwards (perhaps even gas acquired through a merger), which gets frozen on retrograde orbits tilted with the respect to the equatorial plane.

Other possibilities involve a merger scenario (capture of gas that settles in the principle plane forming stars, and/or makes tidal inflows that create KDCs), or growth of a central black hole (Loeb \& Rasio 1994). A black hole stabilizes the disk and within this scenario a connection to quasars can be made by stopping the fueling of the central engines with the formation of a stable disk. None of the previously investigated nuclear stellar disk galaxies has an active nucleus, although they do harbor $10^{8-9} M_{\odot}$ black holes (Kormendy et al. 1996a,b; Cretton \& van den Bosch 1999). This makes them descendants of quasars that spent their fuel (there is not much dust or gas in most of these galaxies), or quasars that, through dynamical evolution, turned off the central engine (stabilization of the disk due to the growth of the black hole, disappearance of bars that transport the material to the center).

Nuclear disks are easier to find in edge-on systems; however, the influence of weak bars is correspondingly more difficult to ascertain. Detailed spectroscopic studies with two dimensional coverage of the major features (nuclei, stellar disks, KDC, photometric features) are necessary to chose between the present formation scenarios. A careful investigation of the twodimensional kinematic properties and their connection to the distribution of line-strengths (metal content and age of stellar populations) can offer decisive tools to deduce the nature and nurture of galaxies with nuclear stellar disks.

Acknowledgements. We are grateful to Michele Cappellari, Eric Emsellem, Richard McDermid, Gijs Verdoes Kleijn, Frank van den Bosch, Zlatan Tsvetanov and Tim de Zeeuw for comments and discussions. D.K. thanks Michele Cappellari and
Harald Kuntschner for making available the pPXF and line-strengths measurement software, respectively. This research has made use of the NASA/IPAC Extragalactic Database (NED) which is operated by the Jet Propulsion Laboratory, California Institute of Technology, under contract with the National Aeronautics and Space Administration. This work also used LEDA database. D.K. was supported by NOVA, the Netherlands Research school for Astronomy.

\section{References}

Bacon, R., Copin, Y., Monnet, G., et al. 2001, MNRAS, 326, 23

Balcells, M., Graham, A. W., Domínguez-Palmero, L., \& Peletier, R. F. 2003, ApJ, 582, L79

Barbon, R., Buondí, V., Cappellaro, E., \& Turatto, M. 1999, A\&AS, 139,531

Barth, A. J., Ho, L. C., \& Sargent, W. L. W. 2002, AJ, 124, 2607

Bender, R. 1988, A\&A, 193, L7

Bender, R. 1990, A\&A, 229, 441

Bender, R., Surma, P., Doebereiner, S., Moellenhoff, C., \& Madejsky, R. 1989, A\&A, 217, 35

Binney, J., \& Tremaine, S. 1987, Galactic dynamics (Princeton, NJ: Princeton University Press), 747

Burkhead, M. S. 1986, AJ, 91, 777

Burstein, D., Faber, S. M., Gaskell, C. M., \& Krumm, N. 1984, ApJ, 287,586

Cappellari, M. 2002, MNRAS, 333, 400

Cappellari, M., \& Emsellem, E. 2004, PASP, 116, 138

Cappellaro, E., Mazzali, P. A., Benetti, S., et al. 1997a, A\&A, 328, 203

Cappellaro, E., Turatto, M., Tsvetkov, D. Y., et al. 1997b, A\&A, 322, 431

Carollo, C. M., Danziger, I. J., Rich, R. M., \& Chen, X. 1997a, ApJ, 491, 545

Carollo, C. M., Franx, M., Illingworth, G. D., \& Forbes, D. A. 1997b, ApJ, 481, 710

Carollo, C. M., Stiavelli, M., \& Mack, J. 1998, AJ, 116, 68

Cohen, J. G. 1985, ApJ, 292, 90

Cretton, N., \& van den Bosch, F. C. 1999, ApJ, 514, 704

Davies, R. L., Efstathiou, G., Fall, S. M., Illingworth, G., \& Schechter, P. L. 1983, ApJ, 266, 41

de Bruyne, V., Vauterin, P., de Rijcke, S., \& Dejonghe, H. 2003, MNRAS, 339, 215

Dolphin, A. E. 2000, PASP, 112, 1397

Emsellem, E., \& Arsenault, R. 1997, A\&A, 318, L39

Emsellem, E., Bacon, R., Monnet, G., \& Poulain, P. 1996, A\&A, 312, 777

Emsellem, E., Dejonghe, H., \& Bacon, R. 1999, MNRAS, 303, 495

Emsellem, E., Monnet, G., \& Bacon, R. 1994, A\&A, 285, 723

Erwin, P., Beltrán, J. C. V., Graham, A. W., \& Beckman, J. E. 2003, ApJ, 597, 929

Erwin, P., \& Sparke, L. S. 1999, ApJ, 521, L37

Erwin, P., \& Sparke, L. S. 2002, AJ, 124, 65

Falcón-Barroso, J., Peletier, R. F., Emsellem, E., et al. 2004, MNRAS, 350, 35

Filippenko, A. V., Porter, A. C., Sargent, W. L. W., \& Schneider, D. P. 1986, AJ, 92, 1341

Fisher, D., Franx, M., \& Illingworth, G. 1996, ApJ, 459, 110

Fisher, D., Illingworth, G., \& Franx, M. 1994, AJ, 107, 160

Freedman, W. L., Madore, B. F., Gibson, B. K., et al. 2001, ApJ, 553, 47

Friedli, D., \& Edry, S. 1993, in Galactic Bulges, IAU Symp., 153, 273

Gerhard, O. E. 1993, MNRAS, 265, 213

González, J. J. 1993, Ph.D. Thesis 
Goudfrooij, P., Hansen, L., Jorgensen, H. E., \& Norgaard-Nielsen, H. U. 1994, A\&AS, 105, 341

Hamuy, M., Philips, M. M., Maza, J., et al. 1994, AJ, 108, 2226

Holtzman, J. A., Burrows, C. J., Casertano, S., et al. 1995, PASP, 107, 1065

Jaffe, W., Ford, H. C., O'Connell, R. W., van den Bosch, F. C., \& Ferrarese, L. 1994, AJ, 108, 1567

Jedrzejewski, R. I. 1987, MNRAS, 226, 747

Kauffmann, G., Guiderdoni, B., \& White, S. D. M. 1994, MNRAS, 267,981

Kimble, R. A., Woodgate, B. E., Bowers, C. W., et al. 1998, ApJ, 492, L83

Kormendy, J. 1988, ApJ, 335, 40

Kormendy, J., \& Bender, R. 1996, ApJ, 464, L119

Kormendy, J., Bender, R., Ajhar, E. A., et al. 1996a, ApJ, 473, L91

Kormendy, J., Bender, R., Richstone, D., et al. 1996b, ApJ, 459, L57

Kormendy, J., Gebhardt, K., Macchetto, F. D., \& Sparks, W. B. 2002, AJ, submitted [arXiv: astro-ph/0107218]

Kregel, M., van der Kruit, P. C., \& de Grijs, R. 2002, MNRAS, 334, 646

Krist, J., \& Hook, R. 2001, The Tiny Tim User's Manual, version 6.0

Kuntschner, H. 1998, Ph.D. Thesis

Kuntschner, H. 2000, MNRAS, 315, 184

Kuntschner, H., Lucey, J. R., Smith, R. J., Hudson, M. J., \& Davies, R. L. 2001, MNRAS, 323, 615

Landsman, W. B. 1993, in Astronomical Data Analysis Software and Systems II, ASP Conf. Ser., 52, 246

Lauer, T. R. 1985, MNRAS, 216, 429

Lauer, T. R., Ajhar, E. A., Byun, Y.-I., et al. 1995, AJ, 110, 2622

Loeb, A., \& Rasio, F. A. 1994, ApJ, 432, 52

Matthews, L. D. 2000, AJ, 120, 1764

Matthews, L. D., Gallagher, J. S., \& van Driel, W. 1999, AJ, 118, 2751

Mehlert, D., Saglia, R. P., Bender, R., \& Wegner, G. 1998, A\&A, 332, 33

Peletier, R. F., Davies, R. L., Illingworth, G. D., Davis, L. E., \& Cawson, M. 1990, AJ, 100, 1091
Pfenniger, D., \& Friedli, D. 1991, A\&A, 252, 75

Pizzella, A., Corsini, E. M., Morelli, L., et al. 2002, ApJ, 573, 131

Rest, A., van den Bosch, F. C., Jaffe, W., et al. 2001, AJ, 121, 2431

Rix, H., \& White, S. D. M. 1990, ApJ, 362, 52

Salvo, M. E., Cappellaro, E., Mazzali, P. A., et al. 2001, MNRAS, 321, 254

Schlegel, D. J., Finkbeiner, D. P., \& Davis, M. 1998, ApJ, 500, 525

Scorza, C., \& Bender, R. 1995, A\&A, 293, 20

Scorza, C., Bender, R., Winkelmann, C., Capaccioli, M., \& Macchetto, D. F. 1998, A\&AS, 131, 265

Scorza, C., \& van den Bosch, F. C. 1998, MNRAS, 300, 469

Seifert, W., \& Scorza, C. 1996, A\&A, 310, 75

Statler, T. 1995, AJ, 109, 1371

Thomas, D., Maraston, C., \& Bender, R. 2003, MNRAS, 343, 279

Trager, S. C., Worthey, G., Faber, S. M., Burstein, D., \& Gonzalez, J. J. 1998, ApJS, 116, 1

Trager, S. C., Faber, S. M., Worthey, G., \& González, J. J. 2000, AJ, 119,1645

Tran, H. D., Tsvetanov, Z., Ford, H. C., et al. 2001, AJ, 121, 2928

van den Bergh, S., \& Younger, P. F. 1987, A\&AS, 70, 125

van den Bosch, F. C., \& de Zeeuw, P. T. 1996, MNRAS, 283, 381

van den Bosch, F. C., \& Emsellem, E. 1998, MNRAS, 298, 267

van den Bosch, F. C., Ferrarese, L., Jaffe, W., Ford, H. C., \& O'Connell, R. W. 1994, AJ, 108, 1579

van den Bosch, F. C., Jaffe, W., \& van der Marel, R. P. 1998, MNRAS, 293, 343

van der Kruit, P. C., Jiménez-Vicente, J., Kregel, M., \& Freeman, K. C. 2001, A\&A, 379, 374

van der Marel, R. P., \& Franx, M. 1993, ApJ, 407, 525

van Dokkum, P. G. 2001, PASP, 113, 1420

Vazdekis, A. 1999, ApJ, 513, 224

Wernli, F., Emsellem, E., \& Copin, Y. 2002, A\&A, 396, 73

Worthey, G. 1994, ApJS, 95, 107

Worthey, G., Faber, S. M., \& Gonzalez, J. J. 1992, ApJ, 398, 69

Worthey, G., Faber, S. M., Gonzalez, J. J., \& Burstein, D. 1994, ApJS, 94,687 
D. Krajnović and W. Jaffe: HST observations of nuclear stellar disks, Online Material $p 1$

\section{Online Material}


D. Krajnović and W. Jaffe: HST observations of nuclear stellar disks, Online Material p 2

Table 2. The summary of HST/WFPC2 observations.

\begin{tabular}{ccccc}
\hline \hline Galaxy & Filter & Date & Time [s] & \# Exp \\
\hline NGC 4128 & $F 450 W$ & 14.05 .2001 & 1400 & 2 \\
& $F 555 W$ & 14.05 .2001 & 800 & 2 \\
NGC 4621 & $F 450 W$ & 14.05 .2001 & 1200 & 2 \\
& $F 555 W$ & 14.05 .2001 & 800 & 2 \\
NGC 5308 & $F 450 W$ & 07.05 .2001 & 1400 & 2 \\
& $F 555 W$ & 07.05 .2001 & 800 & 2 \\
\hline
\end{tabular}

Table 3. The properties of the WFPC2 broad band filters used (from WFPC2 Instrument Handbook), showing the central wavelength and the size of the wavelength window covered by the given filters.

\begin{tabular}{cccc}
\hline \hline Filter & $\lambda_{\text {cen }}[\AA]$ & $\Delta \lambda[\AA]$ & Band \\
\hline F450W & 4410 & 925 & $B$ \\
$F 555 W$ & 5202 & 1223 & $V$ \\
\hline
\end{tabular}

Table 5. Summary of HST/STIS observations.

\begin{tabular}{cccccrr}
\hline \hline Galaxy & Slit & Date & Time & \# Exp & $\Delta_{\text {cen }}$ & PA \\
$(1)$ & $(2)$ & $(3)$ & $(4)$ & $(5)$ & $(6)$ & $(7)$ \\
\hline NGC 4128 & cen & 02.12 .2001 & 2568.5 & $3+3$ & 0.00 & -112.9 \\
& pos & 02.12 .2001 & 2700 & 3 & 0.16 & -112.9 \\
& neg & 02.12 .2001 & 2697 & 3 & -0.40 & -112.9 \\
NGC 4570 & cen & 01.04 .2001 & 2249.5 & $3+3$ & -0.20 & 152.1 \\
& pos & 01.04 .2001 & 2369 & 2 & 0.68 & 152.1 \\
& neg & 01.04 .2001 & 2520 & 2 & -0.56 & 152.1 \\
NGC 4621 & cen & 01.04 .2001 & 2260.5 & $3+3$ & -0.12 & 161.1 \\
& pos & 01.04 .2001 & 2380 & 3 & 0.40 & 161.1 \\
& neg & 01.04 .2001 & 2520 & 3 & -0.44 & 161.1 \\
NGC 5308 & cen & 12.07 .2000 & 2525.0 & $3+3$ & 0.00 & 60.4 \\
& pos & 12.07 .2000 & 2667 & 3 & 0.36 & 60.4 \\
& neg & 12.07 .2000 & 2670 & 3 & -0.36 & 60.4 \\
\hline
\end{tabular}

Notes - Column (1): galaxy name; Col. (2): the position of slit, cen center, pos - positive offset, neg - negative offset (see text for details); Col. (3): date of observations; Col. (4): total exposure time in seconds (exposure time of central slits is the average time of all added observations); Col. (5): number of used observations; Col. (6): distance of the center of the slit from the galaxy nucleus in arcsec; $\mathrm{Col}$ (7): position angle of the slit in degrees east from north.
Table 6. The configuration of STIS and the properties of the grating.

\begin{tabular}{lc}
\hline \hline Quantity & Value \\
\hline Aperture & $52 \times 0.2$ \\
Grating & $\mathrm{G} 430 \mathrm{M}$ \\
$\lambda$-range $[\AA]$ & $5050.4-5381.6$ \\
$\lambda_{\text {cen }}[\AA]$ & 5216 \\
Scale $\Delta \lambda\left[\AA\right.$ pixel $\left.^{-1}\right]$ & 0.28 \\
Spatial scale $\left[\operatorname{arcsec~pixel~}^{-1}\right]$ & 0.05 \\
Comparison line $F W H M[$ pixel] & 2.9 \\
$R=\lambda / \Delta \lambda$ & 6461 \\
Instrumental dispersion $\left[\mathrm{km} \mathrm{s}^{-1}\right]$ & 19.76 \\
\hline
\end{tabular}


D. Krajnović and W. Jaffe: HST observations of nuclear stellar disks, Online Material p 3

Table 7. Bins for kinematic extraction.

\begin{tabular}{|c|c|c|c|c|c|}
\hline Galaxy & Bin & $r$ [arcsec] & Width [pix] & Range & $S / N$ \\
\hline \multirow[t]{12}{*}{ NGC 4128} & Center & 0.00 & 1 & $599-599$ & 15 \\
\hline & $r 1$ & 0.05 & 1 & $600-600$ & 14 \\
\hline & $r 2$ & 0.15 & 2 & $601-603$ & 15 \\
\hline & $r 3$ & 0.25 & 3 & $604-607$ & 13 \\
\hline & $r 4$ & 0.50 & 6 & $608-612$ & 12 \\
\hline & $r 5$ & 1.25 & 24 & $613-637$ & 12 \\
\hline & $l 1$ & -0.05 & 1 & $598-598$ & 14 \\
\hline & 12 & -0.10 & 1 & $596-597$ & 12 \\
\hline & 13 & -0.15 & 2 & $593-595$ & 14 \\
\hline & 14 & -0.30 & 3 & $589-592$ & 13 \\
\hline & 15 & -0.50 & 5 & $583-588$ & 12 \\
\hline & 16 & -0.95 & 13 & $569-582$ & 12 \\
\hline \multirow[t]{12}{*}{ NGC 4570} & Center & 0.00 & 1 & 599-599 & 22 \\
\hline & $r 1$ & 0.05 & 1 & $600-600$ & 21 \\
\hline & $r 2$ & 0.10 & 1 & $601-601$ & 18 \\
\hline & $r 3$ & 0.15 & 2 & $602-604$ & 20 \\
\hline & $r 4$ & 0.30 & 3 & $605-608$ & 20 \\
\hline & $r 5$ & 0.50 & 5 & $609-614$ & 19 \\
\hline & $r 6$ & 0.80 & 8 & $615-623$ & 19 \\
\hline & $l 1$ & -0.05 & 1 & $598-598$ & 19 \\
\hline & $l 2$ & -0.15 & 2 & $595-597$ & 21 \\
\hline & 13 & -0.25 & 3 & $591-594$ & 21 \\
\hline & 14 & -0.45 & 4 & $586-590$ & 19 \\
\hline & 15 & -0.70 & 6 & $579-585$ & 18 \\
\hline \multirow[t]{12}{*}{ NGC 4621} & Center & 0.00 & 1 & $599-599$ & 40 \\
\hline & $r 1$ & 0.05 & 1 & $600-600$ & 34 \\
\hline & $r 2$ & 0.10 & 1 & $601-601$ & 25 \\
\hline & $r 3$ & 0.20 & 2 & $602-604$ & 26 \\
\hline & $r 4$ & 0.35 & 4 & $605-609$ & 27 \\
\hline & $r 5$ & 0.65 & 8 & $610-618$ & 26 \\
\hline & $l 1$ & -0.05 & 1 & $598-598$ & 36 \\
\hline & $l 2$ & -0.10 & 1 & $597-597$ & 28 \\
\hline & 13 & -0.15 & 2 & $594-596$ & 29 \\
\hline & 14 & -0.30 & 3 & $590-593$ & 26 \\
\hline & 15 & -0.50 & 6 & $583-589$ & 27 \\
\hline & $l 6$ & -0.95 & 11 & $571-582$ & 25 \\
\hline \multirow[t]{12}{*}{ NGC 5308} & Center & 0.00 & 1 & $599-599$ & 27 \\
\hline & $r 1$ & 0.05 & 1 & $600-600$ & 23 \\
\hline & $r 2$ & 0.10 & 1 & $601-601$ & 17 \\
\hline & $r 3$ & 0.20 & 2 & $602-604$ & 17 \\
\hline & $r 4$ & 0.30 & 3 & $605-608$ & 15 \\
\hline & $r 5$ & 0.55 & 6 & $609-615$ & 15 \\
\hline & $l 1$ & -0.05 & 1 & $598-598$ & 23 \\
\hline & $l 2$ & -0.10 & 1 & $597-597$ & 17 \\
\hline & 13 & -0.15 & 2 & $594-596$ & 18 \\
\hline & 14 & -0.30 & 3 & $590-593$ & 17 \\
\hline & 15 & -0.50 & 5 & $584-589$ & 16 \\
\hline & 16 & -0.90 & 11 & $572-583$ & 15 \\
\hline
\end{tabular}


D. Krajnović and W. Jaffe: HST observations of nuclear stellar disks, Online Material $p 4$

Table 8. Line-strength indices measured in $0.55 \times 0.0^{\prime} 2$ aperture.

\begin{tabular}{ccccccccccccc}
\hline \hline Index & \multicolumn{3}{c}{ NGC 4128 } & \multicolumn{3}{c}{ NGC 4570 } & \multicolumn{3}{c}{ NGC 4621 } & \multicolumn{3}{c}{ NGC 5308 } \\
slit & cen & neg & pos & cen & neg & pos & cen & neg & pos & cen & -neg & pos \\
\hline $\mathrm{Mg} b$ & $5.0 \pm 0.2$ & - & $5.0 \pm 0.4$ & $4.5 \pm 0.2$ & $2.9 \pm 0.3$ & $3.7 \pm 0.4$ & $5.9 \pm 0.1$ & $6.6 \pm 0.3$ & $4.7 \pm 0.3$ & $4.8 \pm 0.1$ & $5.5 \pm 0.5$ & $5.8 \pm 0.6$ \\
$\mathrm{Fe} 5720$ & $2.9 \pm 0.1$ & - & $2.6 \pm 0.4$ & $3.1 \pm 0.1$ & $1.7 \pm 0.3$ & $1.5 \pm 0.4$ & $3.7 \pm 0.1$ & $3.4 \pm 0.3$ & $3.4 \pm 0.3$ & $3.1 \pm 0.1$ & $4.1 \pm 0.4$ & $3.5 \pm 0.5$ \\
\hline $\mathrm{Mg} b$ & $4.8 \pm 0.2$ & - & $5.0 \pm 0.4$ & $4.4 \pm 0.2$ & $2.9 \pm 0.3$ & $3.5 \pm 0.4$ & $5.6 \pm 0.1$ & $6.4 \pm 0.3$ & $4.6 \pm 0.3$ & $4.7 \pm 0.1$ & $5.3 \pm 0.5$ & $5.7 \pm 0.5$ \\
$\mathrm{Fe} 5720$ & $2.8 \pm 0.1$ & - & $2.6 \pm 0.4$ & $3.1 \pm 0.1$ & $1.7 \pm 0.3$ & $1.4 \pm 0.4$ & $3.6 \pm 0.1$ & $3.4 \pm 0.3$ & $3.3 \pm 0.3$ & $3.0 \pm 0.1$ & $4.0 \pm 0.5$ & $3.5 \pm 0.4$ \\
$B-V$ & 0.97 & - & 0.96 & - & - & - & 0.99 & 1.00 & 1.00 & 0.96 & 0.94 & 0.96 \\
\hline
\end{tabular}

Notes - First two rows in the table present line-strengths (in $\AA$ ) corrected by the velocity dispersion measured using the whole spectral region. The second two rows present line-strengths (in $\AA$ ) corrected by the velocity dispersion measured excluding the $\mathrm{Mg} b$ region from the fit during extraction of kinematics. The last row presents $B-V$ color measured at the actual positions of slits within the same slit-like aperture used for measuring line-strengths. The errors on the color values are estimated to be 0.05 mag. 
D. Krajnović and W. Jaffe: HST observations of nuclear stellar disks, Online Material p 5

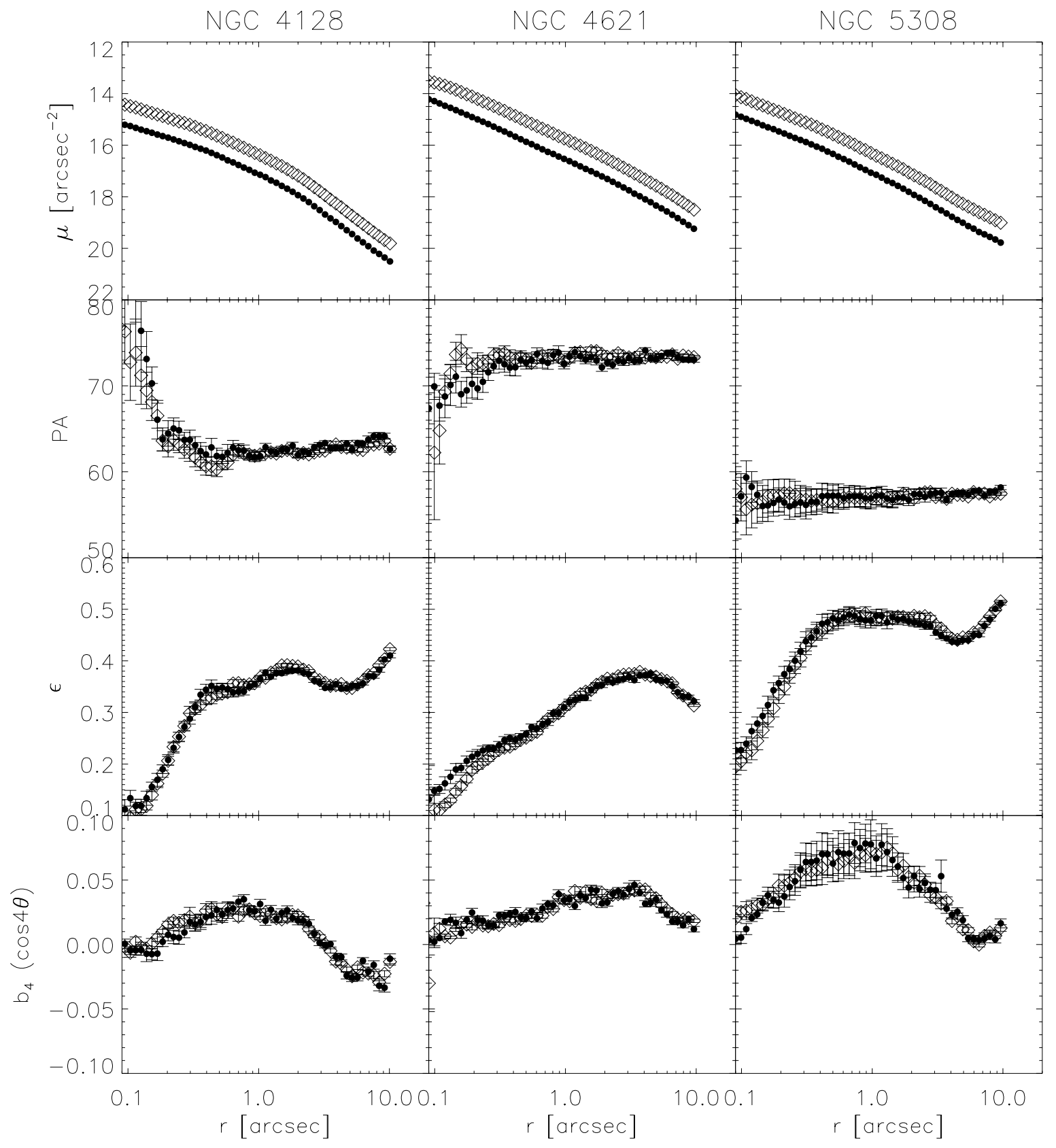

Fig. 1. Isophotal analysis results for NGC 4128, NGC 4621 and NGC 5308 in two observed filters: $B$ (F450W) and V (F555W). First row: surface brightness profiles. Filled symbols correspond to $B$ and open symbols to $V$ filter. Errors are smaller then the symbols. Second row: position angles measured east of north; in the case of NGC 4621 we added $90^{\circ}$ to the measured position angle (east of north) for presentation purposes. Third row: ellipticity. Fourth row: higher order parameters $b_{4}$ (the coefficient of $\cos (4 \theta)$ ), showing deviations from perfect ellipses. 
D. Krajnović and W. Jaffe: HST observations of nuclear stellar disks, Online Material p 6

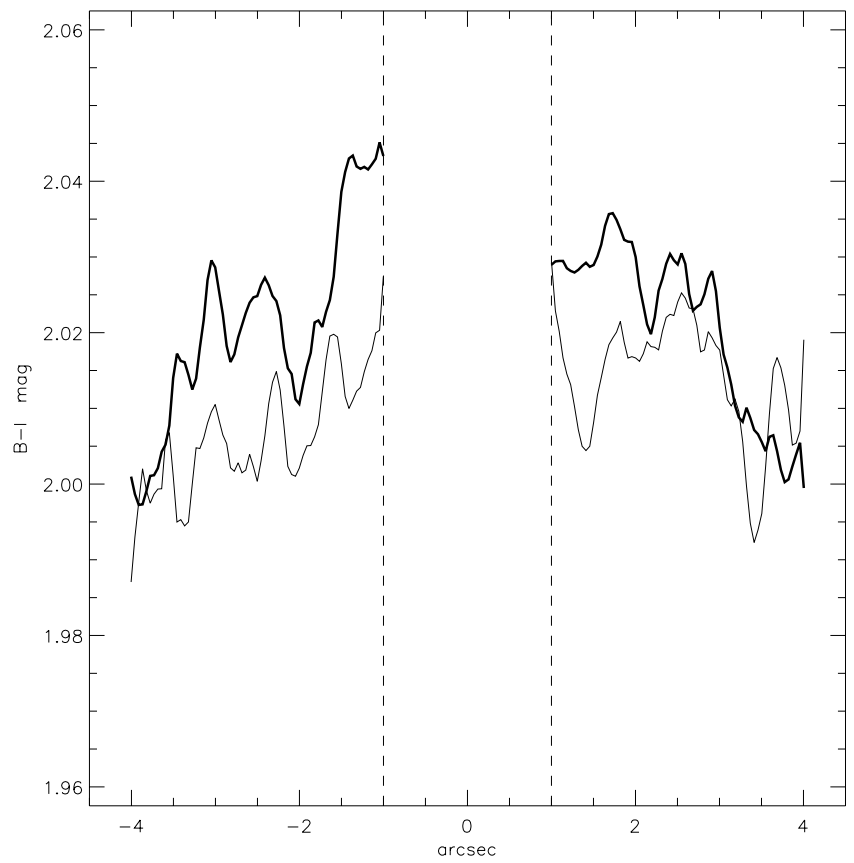

Fig. 3. Comparison of $B-I$ color profiles extracted (and smoothed) along the disk (thin line) and parallel to disk (thick line) in NGC 5308. The slit-like apertures were $200 \times 1$ pixels in size. One slit (thin line) was placed along the major axis (and the disk), while two other slits (averaged together and presented by the thick line) were placed parallel to the major axis, 10 pixels (0'45) above and below it. Vertical dashed lines show the nuclear region excluded from the measurement due to the saturation effects.

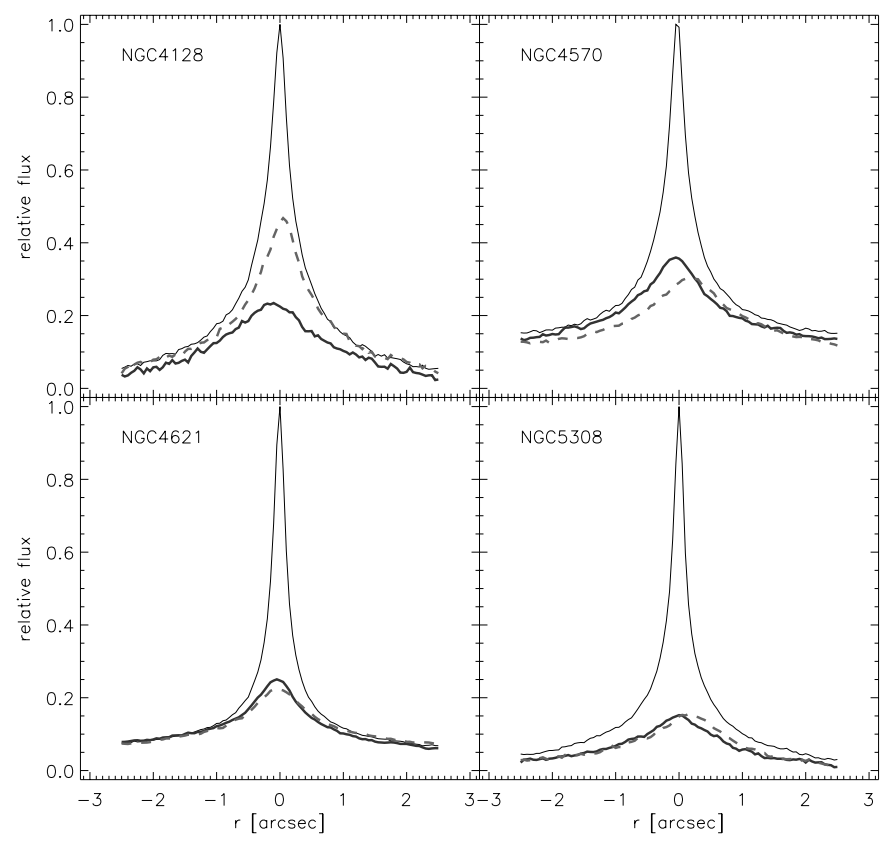

Fig. 4. Galaxy light profile along the STIS slit. The full spectral range was used. Thin dark line is the profile along the cen slit, thick gray lines are profiles along the side slits: dashed line along the pos slit and full line along the neg slit. The profiles were normalized to the maximum of the cen slit in order to emphasize the difference of intensities.

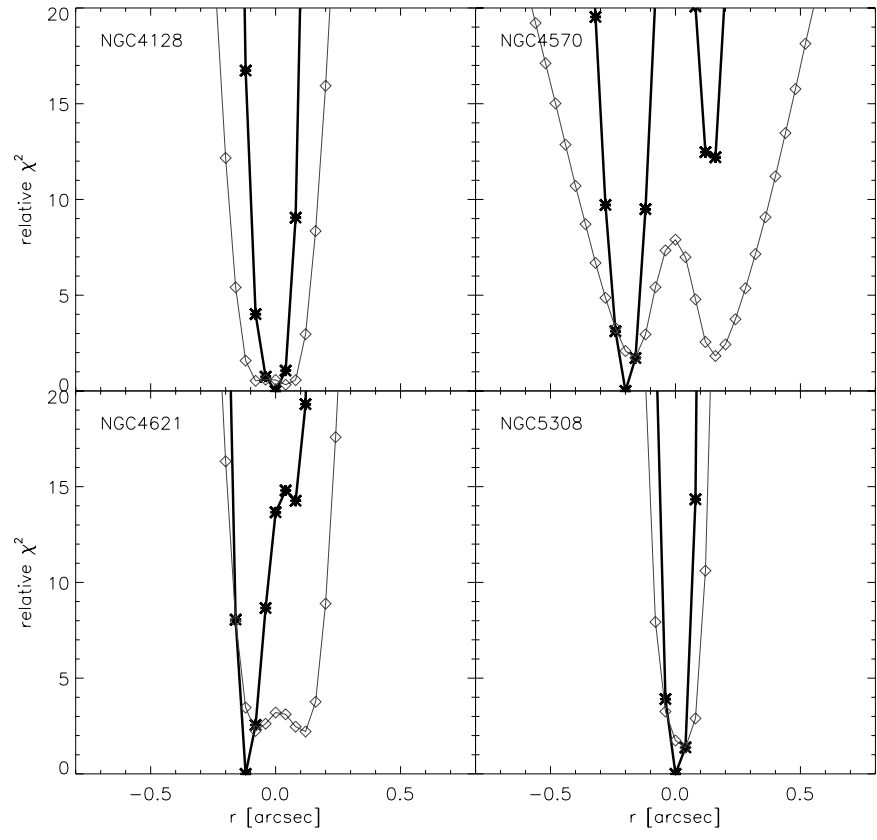

Fig. 5. Plots of $\chi^{2}$ versus the positions of the slits for each galaxy. The line connecting the diamonds is the relative $\chi^{2}$ of the central slit. The line connecting asterisks is the total relative $\chi^{2}$ obtained by including the side slits in calculations.

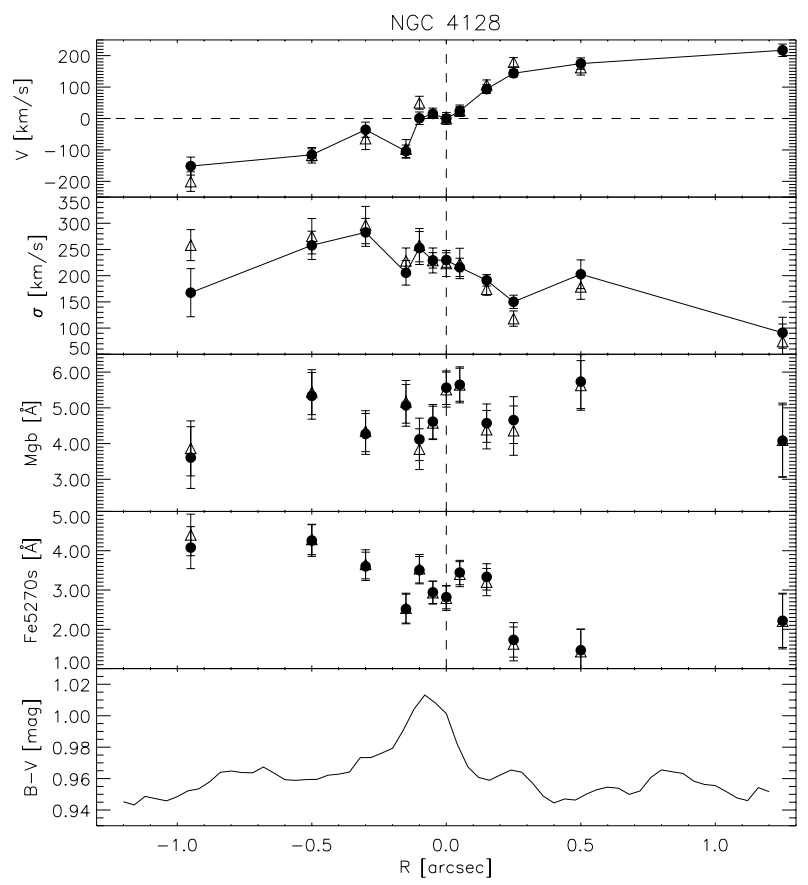

Fig. 8. Kinematic and line-strength profiles for NGC 4128. From top to bottom: mean velocity, velocity dispersion, $\mathrm{Mg} b$ index, Fe5270 index and $B-V$ color profile. The color profile was extracted along the slit position averaging 0 ' 2 perpendicular to the slit. The closed symbols represent measurement obtained by fitting the whole spectral region. The open symbols represent measurement by excluding the $\operatorname{Mg} b$ line from the fit. 
D. Krajnović and W. Jaffe: HST observations of nuclear stellar disks, Online Material p 7

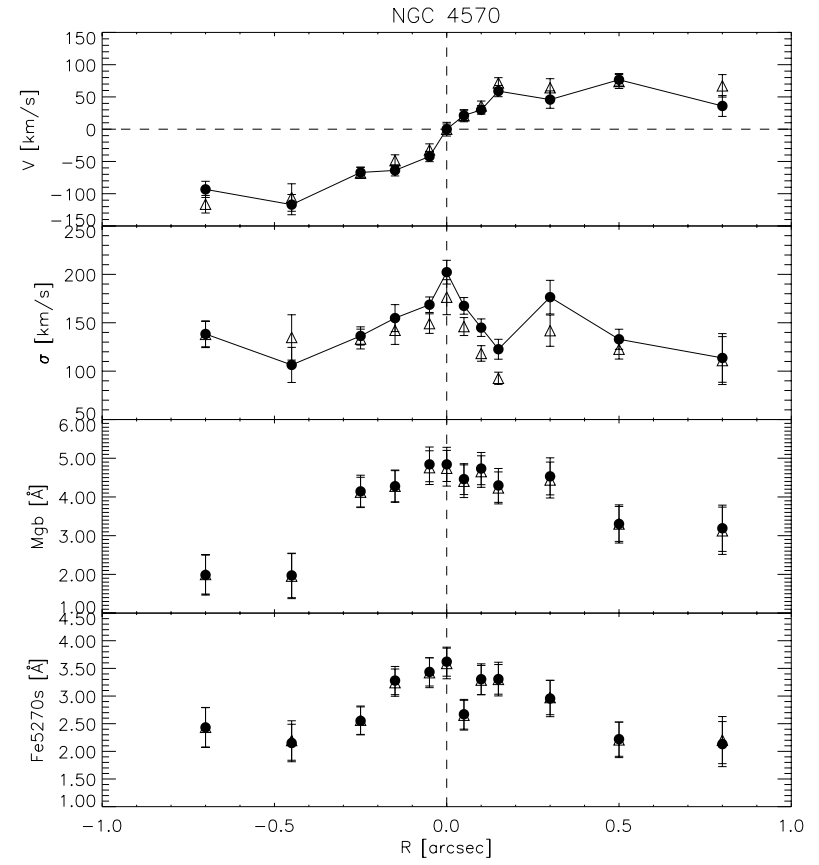

Fig. 9. Kinematic and line-strength profiles for NGC 4570. From top to bottom: mean velocity, velocity dispersion, $\mathrm{Mg} b$ index, $\mathrm{Fe} 5270$ index. The closed symbols represent measurement obtained by fitting the whole spectral region. The open symbols represent measurement by excluding the $\mathrm{Mg} b$ line from the fit.

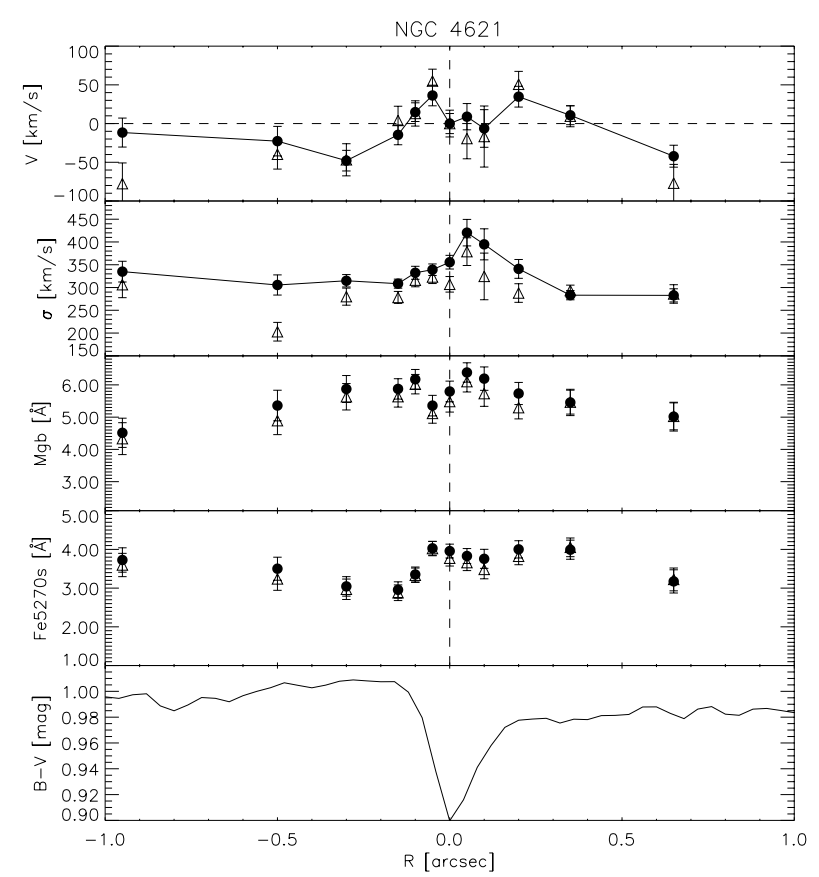

Fig. 10. Kinematic and line-strength profiles for NGC 4621. From top to bottom: mean velocity, velocity dispersion, $\mathrm{Mg} b$ index, Fe5270 index and $B-V$ color profile. The color profile was extracted along the slit position averaging 0 ' 2 perpendicular to the slit. The closed symbols represent measurement obtained by fitting the whole spectral region. The open symbols represent measurement by excluding the $\mathrm{Mg} b$ line from the fit.

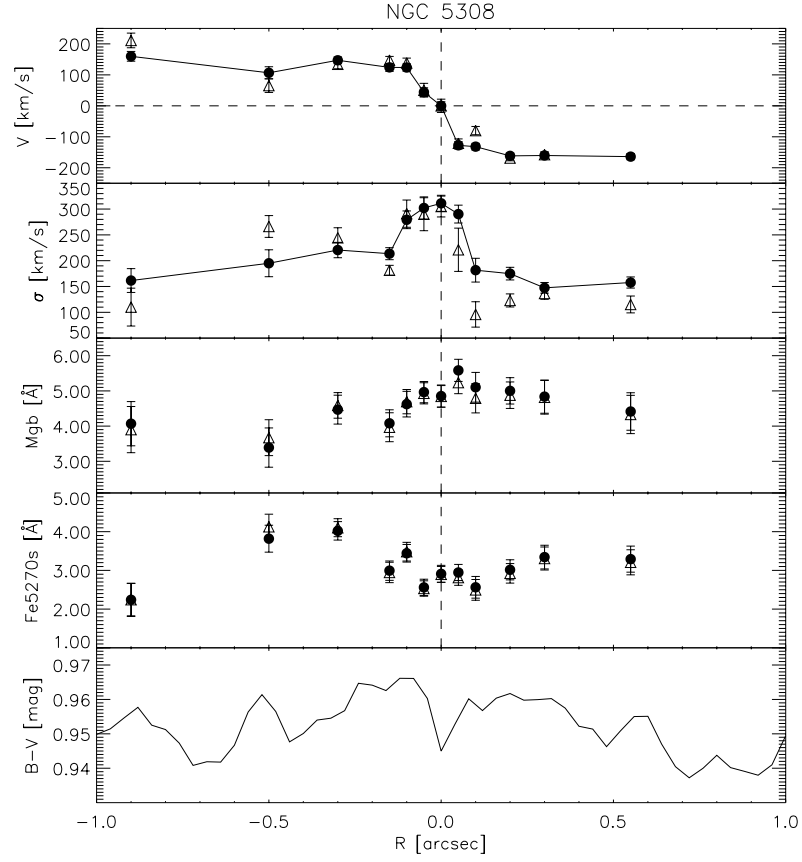

Fig. 11. Kinematic and line-strength profiles for NGC 5308. From top to bottom: mean velocity, velocity dispersion, $\mathrm{Mg} b$ index, Fe5270 index and $B-V$ color profile. The color profile was extracted along the slit position averaging 0.2 perpendicular to the slit. The closed symbols represent measurement obtained by fitting the whole spectral region. The open symbols represent measurement by excluding the $\mathrm{Mg} b$ line from the fit. 

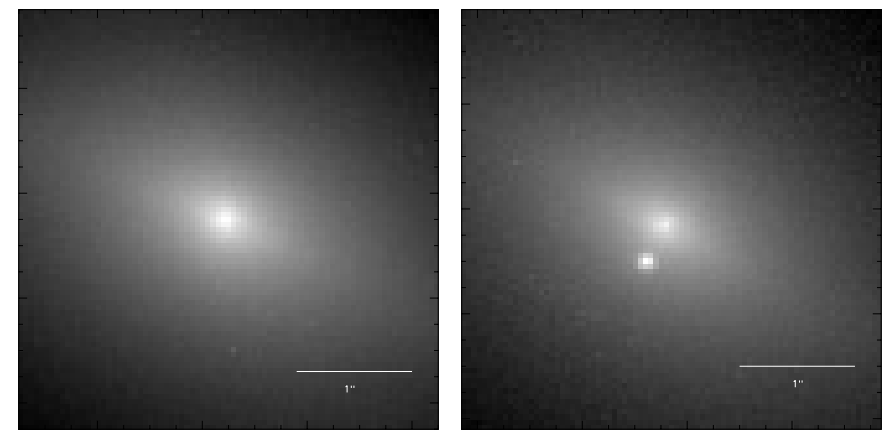

Fig. B.1. Zoom-in on the WFPC2 images of the nucleus of the NGC 4128 , in $F 702 W$ (left) and $F 555 W$ band (right). Both images are oriented as in Fig. 2 (north is down and east to the right). Horizontal line is $1^{\prime \prime}$ long. The left image was taken almost two years before the right one. The offset of the peak next to the center on the right image is $0{ }^{\prime} 143$ west and $0{ }^{\prime} 318$ north from the center of the galaxy.

\section{Appendix B. A transient in NGC 4128}

Examining the HST - WFPC2 images of the nearby S0 galaxy NGC 4128, taken on May 142001 in the F450W and $F 555 W$ filters, a peculiar source of light was discovered very close to the center of the galaxy (Fig. B.1). The source is offset by 0.14 west and 0.32 north of the galaxy center on the WFPC2 images. In the HST archive there are images of the galaxy taken on March 17, 1999, with filter $F 702 W$, observed under PID 6357 (PI Jaffe), which do not show any additional light source next to the nucleus of the galaxy. This clearly indicates the appearance of a transient object in our data. In this Appendix we try to deduce the origin of the transient. Appendix B.1 presents a new method of extraction of the light contribution from the background galaxy and the photometric analysis, followed by discussion on the nature of the object in Appendix B.2.

\section{B.1. Extraction of light and results}

The general properties of NGC 4128 were described in the main text. Here we add that the dust in the galaxy has not been detected (Tran et al. 2001). This ensures there is no significant extinction although the object is almost in the center of the galaxy. The galactic component of the reddening in the direction of the NGC 4128 is estimated to be $E(B-V)=0.02 \mathrm{mag}$ (Schlegel et al. 1998).

To determine the nature of the transient, it is necessary to accurately do photometry on the observed light. The first required step is to remove the contribution of the galaxy light. Since there are no pre-transient observations of the galaxy in the two filters, it was not possible to use the standard technique of subtracting a suitable image of the galaxy taken with the same instrumental set up (e.g. Filippenko et al. 1986; Hamuy et al. 1994). To overcome this problem a Multi-Gaussian Expansion (MGE) model of the galaxy was constructed (e.g. Emsellem et al. 1994). The method and software provided by Cappellari (2002) were used. The MGE models of the galaxies were created using only the $\mathrm{PC} 1$ chip and masking the region of the transient by a circular mask with radius of 5 pixel (Fig. B.2).
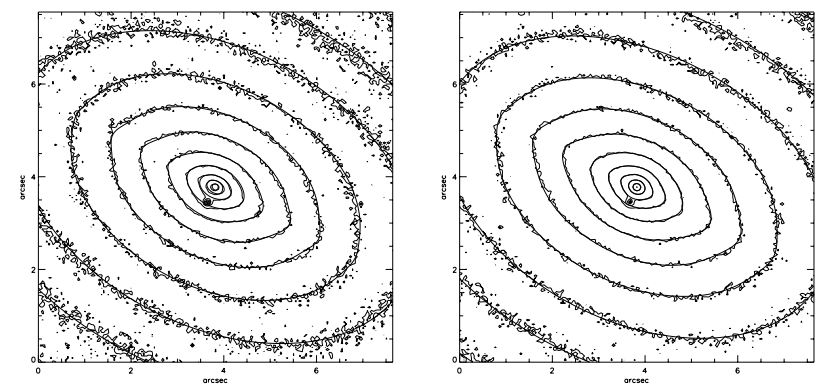

Fig. B.2. Contour maps of WFPC2 F450W (left) and F555W (right) images of NGC 4128. The images show the inner 7".5. Superposed on the two images are the contours of the MGE surface brightness, convolved with the WFPC2 PSFs for the corresponding filters. The transient was excluded from the fit, but it is shown in the images for illustration.
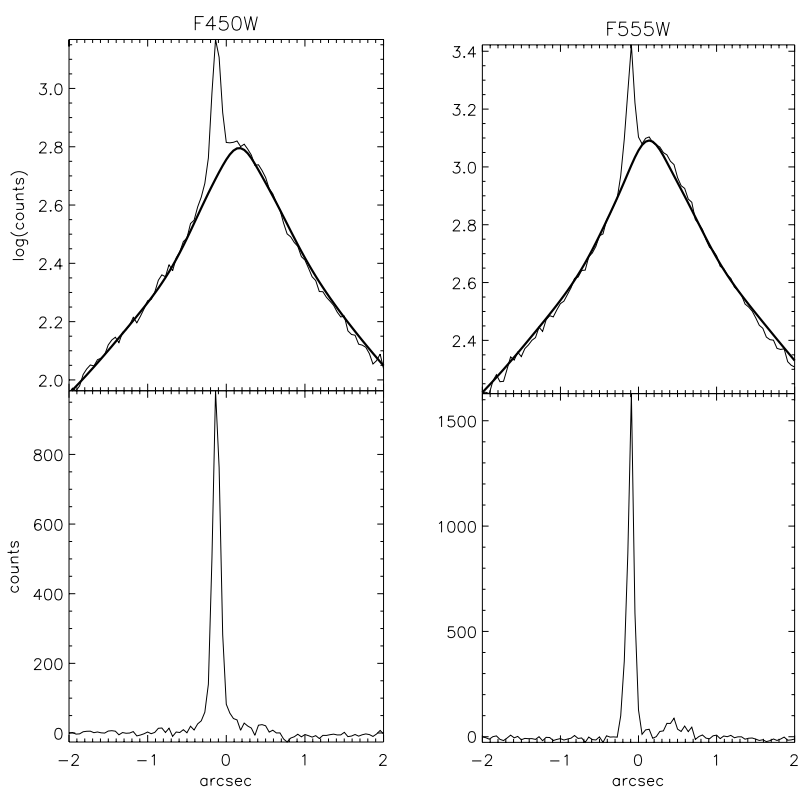

Fig. B.3. Horizontal cuts through the galaxy on the position of the SN. The thick line is the MGE model and the thin line is the observed light. The lower plots show residuals obtained subtracting the models from the observations. The transient is clearly separable from the rest of the galaxy by a MGE model.

For both MGE models, the total number of used Gaussians was increased until the minimum $\chi^{2}$ stopped decreasing. This approach yielded the total number of 9 Gaussians and RMS error of about $2 \%$.

The obtained models were subtracted form the original images. Figure B.3 shows a horizontal cut through the observed image at the position of the transient and the corresponding MGE model. The light of the transient is clearly distinguishable from the rest of the galaxy. Subtracting the MGE model of the galaxy from the observations it is possible to recover the transient light contribution. The residuals are symmetric and can be used to calculate the magnitude of the transient. Photometric measurements were conducted using the IRAF task PHOT. Magnitude zero points for filters were obtained from Dolphin (2000). The values calculated in an aperture of 0.5 were corrected to infinite aperture by adding $0.1 \mathrm{mag}$. 
Table B.1. Summary of transient measurements.

\begin{tabular}{ccccccccc}
\hline \hline Filter & RA & Dec & $\delta x$ & $\delta y$ & Counts & $m$ & $\sigma_{m}$ & $M$ \\
$(1)$ & $(2)$ & $(3)$ & $(4)$ & $(5)$ & $(6)$ & $(7)$ & $(8)$ & $(9)$ \\
\hline$F 450 W$ & $12: 08: 34.84$ & $68: 46: 57.15$ & 0.142 & 0.315 & 20940.09 & 19.06 & 0.05 & -13.7 \\
$F 555 W$ & $12: 08: 34.84$ & $68: 46: 57.25$ & 0.143 & 0.318 & 18099.38 & 19.17 & 0.05 & -13.6 \\
\hline
\end{tabular}

(1): Name of the filter; (2) and (3): position of the transient measured in (h, m, s) and (deg, arcmin, arcsec) on WFPC2 (J2000); (4) and (5): the offset in arcsec of the transient from the intensity peak of the galaxy due west and north respectively; (6) total counts inside aperture of 0 '.5; (7) magnitude of the transient in Johnson-Cousins system; (8) the estimated error on the apparent magnitude; (9) absolute magnitude using the distance to NGC 4128 from Table 1.

The correction for geometrical distortion and the CTE correction were not performed as their influence are of second order, compared to the uncertainty introduced by subtracting the model galaxy from the original image. The final transient parameters, namely its position, apparent magnitude and absolute magnitude in Johnson-Cousins system, are given in the Table B.1.

\section{B.2. Discussion and conclusions}

Lacking any spectra of the detected object as well as a longer period of observation, it is difficult to determine the object's true nature. Since it is not visible on the $F 702 \mathrm{~W}$ band image while it is quite bright on the $F 450 \mathrm{~W}$ and $F 555 \mathrm{~W}$ band images (taken 22 months later), it is most natural to conclude the object is a transient. There are a few possibilities such as a solar system object, a nova or a supernova.

The duration of all four independent WFPC2 exposures is about $40 \mathrm{~min}$. A solar system object would show a noticeable movement between the different exposures, except if moving directly toward an observer. If the object is at the distance of the Kuiper belt it would move approximately a few arc seconds during the observations. However, the relative position of the object (the distance between the object and the center of the galaxy) changes by less than one pixel $(<0$ ' 0455$)$, the movement being less than measurement error. It seems reasonable to conclude the object is of extra-Solar system origin.

Novae are known to have a maximum absolute magnitude less bright then -9 mag (Cohen 1985). With the measured absolute magnitude of -13.6 ( $V$ band), this is ruled out. Similarly, because it is very faint, the observed transient is also not likely a nova in our galaxy (a compilation of novae light curves is given in van den Bergh \& Younger 1987). The observed magnitude leaves the possibility that the object in NGC 4128 is a supernova. The type of supernova is defined according to its spectrum, and here again it is hard to establish anything specific. However, the host galaxy is an S0 galaxy, and since supernovae of type II do not occur in early-type galaxies (Table 3, in Cappellaro et al. 1997b), the transient is probably a supernova of type Ia. Comparing the measured absolute magnitude to absolute magnitudes of supernova 1994D or 1992A (Cappellaro et al. 1997a), which were also S0 galaxies, suggests that the supernova was observed about 200 days after the explosion. The $B-V$ color of the supernova is -0.11 , which is bluer then the expected color for a type Ia $\mathrm{SN}$ at $\mathrm{B}$ maximum. On the other had, it is consistent with the $B-V$ light curve at the later time (around 200 days) of the above mentioned reference supernovae (Salvo et al. 2001).

Private communication with the supernova-survey groups (D. Green from IAU Circulars and W. Li from LOTOSS survey) did not confirm the existence of the supernova. They checked images from January to June 2001, and after galaxy subtraction there was no signature of any transient object. However, the proximity to the nucleus of NGC 4128 is a possible reason why ground-based automated search program might have overlooked the supernova.

If the source of the light is a type Ia supernova, it will be the first supernova discovered in NGC 4128 (Barbon et al. 1999) 4 Unfortunately, without other high resolution available data it is impossible to determine the true nature of the object. Also, the estimate of the timing of the supernova in NGC 4128 is highly uncertain, since it depends on precise classification and calibration. Type Ia supernovae are known to have different absolute magnitude light curves (e.g. Cappellaro et al. 1997a). If the transient in the NGC 4128 is not a supernova of type Ia 200 days after explosion then its nature remains unknown (a flaring second black hole, an asteroid on the trajectory to Earth). 